\title{
A pseudo-spectral multiscale method: Interfacial conditions and coarse grid equations
}

\author{
Shaoqiang Tang ${ }^{\mathrm{a}, \mathrm{b}}$, Thomas Y. Hou ${ }^{\mathrm{b}, *}$, Wing Kam Liu ${ }^{\mathrm{c}}$ \\ ${ }^{a}$ LTCS, Department of Mechanics and Engineering Science, Peking University, Beijing 100871, PR China \\ ${ }^{\mathrm{b}}$ Applied and Computational Mathematics, California Institute of Technology, 217-50, Caltech, Pasadena, CA 91125, United States \\ ${ }^{\mathrm{c}}$ Department of Mechanical Engineering, Northwestern University, Evanston, IL 60208, United States
}

Received 28 March 2005; received in revised form 31 July 2005; accepted 1 August 2005

Available online 12 September 2005

\begin{abstract}
In this paper, we propose a pseudo-spectral multiscale method for simulating complex systems with more than one spatial scale. Using a spectral decomposition, we split the displacement into its mean and fluctuation parts. Under the assumption of localized nonlinear fluctuations, we separate the domain into an MD (Molecular Dynamics) subdomain and an MC (MacrosCopic) subdomain. An interfacial condition is proposed across the two scales, in terms of a time history treatment. In the special case of a linear system, this is the first exact interfacial condition for multiscale computations. Meanwhile, we design coarse grid equations using a matching differential operator approach. The coarse grid discretization is of spectral accuracy. We do not use a handshaking region in this method. Instead, we define a coarse grid over the whole domain and reassign the coarse grid displacement in the MD subdomain with an average of the MD solution. To reduce the computational cost, we compute the dynamics of the coarse grid displacement and relate it to the mean displacement. Our method is therefore called a pseudo-spectral multiscale method. It allows one to reach high resolution by balancing the accuracy at the coarse grid with that at the interface. Numerical experiments in one- and two-space dimensions are presented to demonstrate the accuracy and the robustness of the method.
\end{abstract}

(c) 2005 Elsevier Inc. All rights reserved.

Keywords: Multiscale method; Interfacial conditions; Coarse grid equations

\section{Introduction}

Multiple spatial scales are encountered in many complex physical problems of importance. Rapid progress in nano-science and nano-technology triggers intensive studies in developing effective

\footnotetext{
* Corresponding author.

E-mail address: hou@acm.caltech.edu (T.Y. Hou).
} 
multiscale models and numerical methods for these problems. A number of multiscale computational methods have been developed to model multiscale materials, including the quasicontinuum method to simulate static problems [24], the coarse-grained molecular dynamics method [22], the bridging scale method [28,15], and the "macroscopic, atomistic, ab initio dynamics" method [4] to simulate the dynamics.

For a typical multiscale computation, one performs Molecular Dynamics (MD) computation only in the localized region where the atomistic description is necessary to model the underlying physics properly, while a coarse grid description is used in the surrounding region. The major difficulties with this class of method are:

- The derivation of the governing equations for the coarse grid dynamics.

- The passage of information between the atomistic region and the coarse grid region.

In particular, it is well known that the abrupt termination of the atomistic region leads to strong spurious wave reflections at the interface between the two scales [28]. Due to the interaction between two regions, these reflections spread errors to the whole domain. Extensive research has been conducted in an attempt to reduce these numerical reflections of waves. Because the atomistic displacements include various wave numbers, the speed of propagation of the high frequency waves depends on their wave numbers via the discrete dispersion relationship. Thus, traditional non-reflecting boundary conditions derived for wave propagations in homogeneous media cannot be applied here [9-12].

For most multiscale methods, a handshaking region is introduced to reduce interfacial reflections $[2,4,14]$. In this region, one either uses a certain weighted average between the MD description and the coarse grid description, or an artificial damping to diffuse the outgoing fluctuations [3]. Unfortunately, these methods are usually proposed in an heuristic manner, and do not accurately describe fluctuations across the interfaces. The hybrid method [7,18] and the dynamic atomistic-continuum method [8] encounter the same difficulty.

In this study, we are concerned with atomistic displacements governed by the Newton laws (a system of coupled ordinary differential equations), under the assumption that atomistic description is only necessary in a localized region containing the defect of the material. We will use a full MD computation in the localized region containing the defect. We also perform a MacrosCopic (MC) computation with a coarse grid over the whole domain, at a much lower computational cost. Motivated by the bridging scale method (BSM) $[13,16,20,28]$, we develop a pseudo-spectral multiscale method with balanced accuracy on coarse and fine scales, as well as at the interfaces. More precisely, by a spectral decomposition, we split the displacements into the mean part and fine fluctuation part. This helps to reduce energy interchange between these two parts across the interfaces. Together with a time history treatment, we accurately reconstruct displacements at the ghost point atoms, which in turn provide interfacial conditions for the MD computations. In the case of linear systems, our method makes no approximation up to this point, and provides the first exact interfacial condition for multiscale computations. In practice, we make use of a partial time history convolution. To further reduce the computing load, the mean displacements are approximated by interpolating the displacements at the coarse grid. Our method is therefore of a pseudo-spectral type. We further propose a matching differential operator method to derive the coarse grid equations with arbitrary desired order of accuracy, up to a spectral resolution of the selected coarse grid. We do not use a handshaking region here. The mean displacements in the MD region are reassigned by averaging the MD solution.

We remark that the additional computational cost is reasonable. The resolution of the pseudo-spectral multiscale method is considerably enhanced compared with other multiscale methods such as the BSM method. Moreover, with a fixed domain decomposition and coarsening ratio, the mathematical formulation of the pseudo-spectral multiscale method gives a systematic derivation of the interfacial conditions and the coarse grid equations. 
There are several advantages of the the pseudo-spectral multiscale method, compared with other existing multiscale computational methods.

- An arbitrary error reduction is obtained due to the accurate time history treatment and coarse grid equations derived by the matching differential operator method. This is a major advantage of the pseudo-spectral multiscale method compared with other multiscale methods, which rely heavily on empirical derivations due to lack of a systematic mathematical analysis. We demonstrate this nice feature, using several detailed numerical experiments in both one and two space dimensions.

- The interfacial reflection reduction is robust, insensitive to the size of the MD region. For lattices in one space dimension, the interfacial reflection is effectively reduced, with an amplitude about one tenth that of a BSM scheme.

- Using the coarse grid equations designed by the matching differential operator method, we capture correct propagation speed for long waves in the coarse grid.

- The pseudo-spectral multiscale method applies to complex situations including high space dimensions and non-nearest neighbor interactions. In particular, we simulate a two-dimensional fracture problem with a relatively thin MD region, and successfully resolve not only the tip position, but also the detailed profile in both the MD region and the coarse grid accurately.

The rest of this paper is organized as follows. We shall formulate the the pseudo-spectral multiscale method in Sections 2 and 3, particularly the interfacial conditions and coarse grid equations. The general framework is illustrated through several interesting applications, including a linear system (harmonic lattice), a nonlinear anharmonic lattice, and a lattice with the Lennard-Jones potential in one space dimension. We also demonstrate the performance of the method using a two-dimensional example of a Slepyan fracture model. A flowchart for the method is presented in Section 4. The numerical results are shown in Section 5. In the last section, we make some concluding remarks.

\section{Displacements and domain decompositions, interfacial conditions}

Consider a solid body in $\Omega \subset \mathbb{R}^{3}$, consisting of $n_{a}$ atoms. The position of the $n$th atom at rest is $x_{n}$. Let $u, f, f_{\text {ext }} \in \mathbb{R}^{3 n_{a}}$ represent the displacement, internal force and external force, respectively. Motion of the atoms is governed by the Newton law

$$
M_{A} \ddot{u}=f+f_{\mathrm{ext}} .
$$

Here the mass matrix is $M_{A}=\operatorname{diag}\left(m_{1} I_{3 \times 3}, \ldots, m_{n_{a}} I_{3 \times 3}\right)$, with $m_{n_{i}}>0$. The internal force comes from atomistic interactions, described by a potential $U$ as

$$
f=-\nabla_{u} U(u)
$$

\subsection{Decomposition of the displacements and computational domains}

To decompose the motion into two scales, we specify a symmetric matrix $L_{3 n_{a} \times 3 n_{a}}$. The choice of $L$ will be discussed later. We require that there exist a complete set of normalized eigenvectors $\Psi_{j} \in \mathbb{R}^{3 n_{a}}$ for the generalized eigenvalue problem: 


$$
L \Psi_{j}=\eta_{j} M_{A} \Psi_{j}, \quad \Psi_{i}^{\mathrm{T}} M_{A} \Psi_{j}= \begin{cases}1, & i=j, \\ 0, & i \neq j .\end{cases}
$$

We refer to each eigenvector as a mode. A characteristic wavelength is recognized for each mode. We select $3 n_{\mathrm{c}}$ lowest order modes to form $N=\left(\Psi_{1}, \ldots, \Psi_{3 n_{\mathrm{c}}}\right)$ and let $H=\operatorname{diag}\left(\eta_{1}, \ldots, \eta_{3 n_{\mathrm{c}}}\right)$.

The optimal linear combination of these modes to represent $u$ is obtained by minimizing a residual functional $(u-N \tilde{d})^{\mathrm{T}} M_{A}(u-N \tilde{d})$. The solution is

$$
\tilde{d}=N^{\mathrm{T}} M_{A} u \text {. }
$$

Its components are the amplitudes of the $3 n_{\mathrm{c}}$ lowest order modes in $u$. We further define

$$
\bar{u}=N \tilde{d}=N N^{\mathrm{T}} M_{A} u \equiv P u, \quad u^{\prime}=(I-P) u \equiv Q u,
$$

where $\bar{u}$ is a low order mode approximation of $u$, and $u^{\prime}$ represents the remaining high order residual. We refer to them as the mean displacements and fine fluctuations, respectively.

From (3), it can be shown that

$$
L N=M_{A} N H, \quad M_{A} Q=Q^{\mathrm{T}} M_{A}, \quad L Q=Q^{\mathrm{T}} L .
$$

Let $A(u)=f(u)-L u$. The low order mode amplitude $\tilde{d}$ evolves according to

$$
\begin{aligned}
\ddot{\tilde{d}} & =N^{\mathrm{T}}\left(L u+A(u)+f_{\text {ext }}\right) \\
& =H \tilde{d}+N^{\mathrm{T}}\left(A(u)+f_{\text {ext }}\right) .
\end{aligned}
$$

The fine fluctuation equation reads

$$
\begin{aligned}
M_{A} \ddot{u}^{\prime} & =Q^{\mathrm{T}}\left(L u+A(u)+f_{\text {ext }}\right) \\
& =L u^{\prime}+Q^{\mathrm{T}}\left(A(u)+f_{\text {ext }}\right) .
\end{aligned}
$$

In many applications, nonlinear interactions among different scales are active only in certain localized regions. In such regions, we must perform detailed MD computations to resolve the physics. We denote the collection of these regions as $\Omega_{\mathrm{D}}$. On the other hand, the mean displacement description is satisfactory for the complimentary subdomain $\Omega_{\mathrm{C}}=\Omega \backslash \Omega_{\mathrm{D}}$.

In the following, we shall extensively use subscript $\mathrm{D}$ to denote quantities in $\Omega_{\mathrm{D}}$, and $\mathrm{C}$ for those in $\Omega_{\mathrm{C}}$. For instance, the displacement vector $u$ and the matrix $L$ are

$$
u=\left[\begin{array}{l}
u_{\mathrm{D}} \\
u_{\mathrm{C}}
\end{array}\right], \quad L=\left[\begin{array}{ll}
L_{\mathrm{DD}} & L_{\mathrm{DC}} \\
L_{\mathrm{CD}} & L_{\mathrm{CC}}
\end{array}\right] .
$$

In the pseudo-spectral multiscale method, we adopt the multiscale methodology to restrict the MD computation in $\Omega_{\mathrm{D}}$, and to perform an MC computation to obtain the mean displacement in $\Omega_{\mathrm{C}}$. The MC computation is actually performed for a coarse grid variable $d$ over the whole domain $\Omega$, which has a much smaller degree of freedom than $u$. The computing cost is considerably reduced in this way.

However, two new challenges emerge in this approach. The first challenge is to compute the mean displacement. In particular, we need to approximate the effects of the fine fluctuations on the mean displacement. Because we do not compute the fine fluctuation $u_{\mathrm{C}}^{\prime}$ in $\Omega_{\mathrm{C}}, A(u)$ needs to be approximated in Eq. (7). Derivation of accurate and efficient coarse grid equations is crucial for multiscale algorithm. The second challenge comes from the domain decomposition. The abrupt termination of the atomistic region leads to strong spurious wave reflections at the interface between the two scales [28]. These spurious reflections can be amplified through the strong nonlinear interactions in $\Omega_{\mathrm{D}}$. Thus the interesting physics in the MD region can be reproduced only when the spurious reflections are considerably reduced. We will address these two challenging issues in Section 3 and the next subsection, respectively. 


\subsection{Interfacial conditions}

Most multiscale methods reduce interfacial reflection by making use of a handshaking region, where either a weighted average is performed between the MD description and the coarse grid description, or an artificial damping is applied [3,4,22]. Usually proposed in an heuristic manner, these methods have a limited accuracy in describing fluctuations across the interfaces. On the other hand, using macroscopic equations (e.g., Navier-Stokes equations) for the coarse grid, special interfacial treatments were proposed, such as a hybrid method in [7,18] and a dynamic atomistic-continuum method in [8]. These conditions cannot cleanly damp out the reflections without making over-damping on physical motions, when a broadband of wave numbers are present. In the literature, there is also a class of non-reflecting boundary conditions and Dirichlet-to-Neumann boundary conditions for scattering problems or exterior wave problems [9-12]. These techniques do not apply to our study here because we are dealing with a discrete lattice and the wave speed of the atomistic displacements depends on the wave numbers.

Another interfacial treatment assumes linearity along a semi-infinite periodic chain. Based on the study of a harmonic lattice [1], the exact displacement of the first atom outside of the main MD region may be expressed as a convolution of the time history at the interfacial atom [5]. By further including the idea of separation of the displacement into a mean and a fluctuation part, the bridging scale method (BSM) uses the time convolution only for the fluctuation part $[25,27,28]$.

Due to its clear and precise form, we use the time history treatment as a starting point in developing our multiscale method. We notice that the internal force on an interfacial atom in $\Omega_{\mathrm{D}}$ involves the displacement at certain neighboring ghost point atoms in $\Omega_{\mathrm{C}}$. The interfacial condition amounts to a reconstruction of displacement $u_{\mathrm{G}}$ at these ghost point atoms. This includes two steps, namely, to calculate the mean displacement and to find the fine fluctuation. We compute the mean displacement by MC computation and interpolation described in Section 3. Here we focus on the fine fluctuation $u_{\mathrm{G}}^{\prime}$.

For this purpose, we apply the Laplace transform to a subsystem of (8)

$$
M_{A_{\mathrm{C}}} \ddot{u}_{\mathrm{C}}^{\prime}=L_{\mathrm{CC}} u_{\mathrm{C}}^{\prime}+L_{\mathrm{CD}} u_{\mathrm{D}}^{\prime}+Q_{\mathrm{C}}^{\mathrm{T}}\left(A(u)+f_{\mathrm{ext}}\right) .
$$

By straightforward manipulations, we find that

$$
\widehat{u_{\mathrm{C}}^{\prime}}=\left(s^{2} I-M_{A_{\mathrm{C}}}^{-1} L_{\mathrm{CC}}\right)^{-1}\left[M_{A_{\mathrm{C}}}^{-1}\left(L_{\mathrm{CD}} \widehat{u_{\mathrm{D}}^{\prime}}+Q_{\mathrm{C}}^{\mathrm{T}}\left(\widehat{A(u)}+\hat{f}_{\mathrm{ext}}\right)\right)+s u_{\mathrm{C}}^{\prime}(0)+\dot{u}_{\mathrm{C}}^{\prime}(0)\right] \text {. }
$$

In general, this is a nonlinear integral equation, because $A(u)$ depends on $u_{\mathrm{C}}^{\prime}$. If we choose $L=\nabla_{u} f=-\nabla_{u} \otimes \nabla_{u} U(u)$, then $A(u)$ contains only nonlinear terms with respect to $u_{\mathrm{C}}$. Based on the assumption of localized nonlinear fluctuations, we further approximate (11) at ghost point atoms by ignoring the nonlinear term $A(u)$. More precisely, for each ghost point atom, we take the corresponding row from the time history kernel matrix

$$
\widetilde{\Phi}=\mathscr{L}^{-1}\left[\left(s^{2} I-M_{A_{\mathrm{C}}}^{-1} L_{\mathrm{CC}}\right)^{-1}\right] .
$$

Let us denote by $\widetilde{\Phi}_{\mathrm{G}}(t)$ the submatrix formed by these rows. We get $u_{\mathrm{G}}^{\prime}$ from the following formula:

$$
u_{\mathrm{G}}^{\prime} \approx \widetilde{\Phi}_{\mathrm{G}}(t) *\left[M_{A_{\mathrm{C}}}^{-1}\left(L_{\mathrm{CD}} u_{\mathrm{D}}^{\prime}+\widetilde{Q}_{\mathrm{C}}^{\mathrm{T}} f_{\mathrm{ext}}\right)\right]+\dot{\widetilde{\Phi}}_{\mathrm{G}}(t) u_{\mathrm{C}}^{\prime}(0)+\widetilde{\Phi}_{\mathrm{G}}(t) \dot{u}_{\mathrm{C}}^{\prime}(0)
$$

The atomistic internal force decreases quickly with respect to the interatomic distance. Accordingly, $L$ and hence $L_{\mathrm{CC}}$ are sparse matrices with a few off-diagonal entries. This results in a relatively simple form of $\widetilde{\Phi}_{\mathrm{G}}(t)$.

We make a few remarks here. First, if the system is linear, the expression (13) is exact. This is in contrast with other interfacial conditions for multiscale algorithms, which are not exact for lattice structures. 

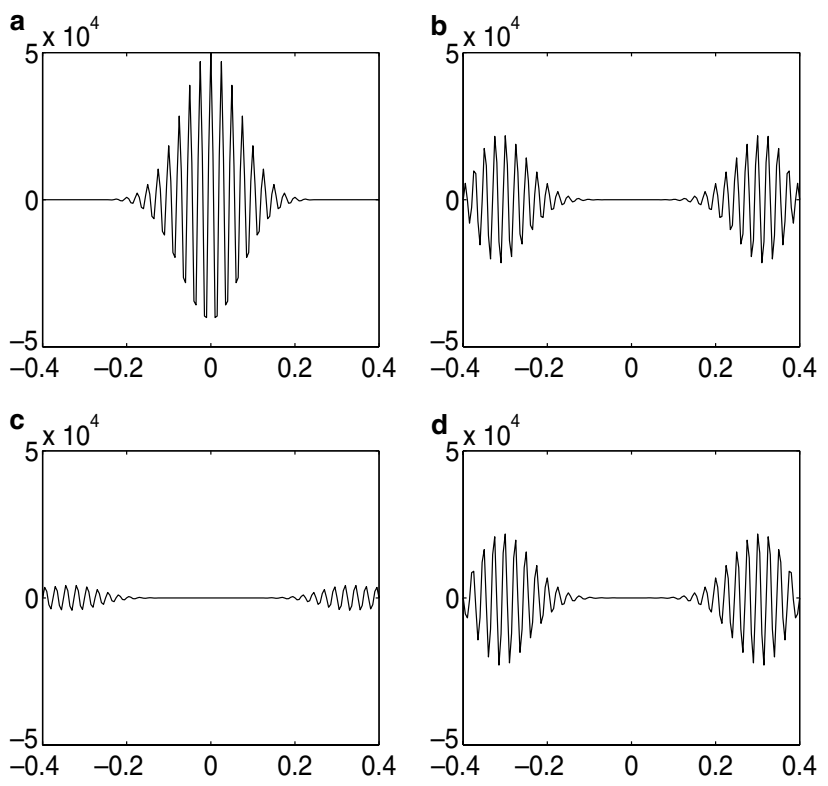

Fig. 1. Reflection in a harmonic lattice for initial fluctuation of a magnitude 0.0005 (atomic distance is 0.005 ), computed with the interfacial condition proposed in [8], yet without a moving mesh treatment: (a) $t=0$; (b) $t=80$; (c) $t=99$ and (d) $t=120$.

For instance, the interfacial conditions proposed in [8] will give reflections that come back after being absorbed, if one does not incorporate a moving mesh strategy. This is shown for a harmonic lattice in Fig. 1. Under our assumption of localized nonlinear fluctuations, in theory, interfacial reflection can be completely eliminated if we can maintain a full record of the displacement time history and use an exact convolution. In practice, one often compromises between computational expense and accuracy, and uses a partial time history of displacement. Secondly, for general systems, the nonlinear effects decrease with the decrement of $\left\|u_{\mathrm{C}}^{\prime}\right\|$. Thirdly, when $n_{a} \rightarrow+\infty$, it is virtually impossible to numerically find all modes for a general $L$. However, in the case of repeated structures, we may select $L$ in a special form such that the modes can be computed theoretically. The time history treatment was adopted for a multiscale computation in BSM [28]. However, with a coarse-fine decomposition based on the linear finite element interpolation, BSM produces a complicated time history kernel matrix, and an approximation is implicitly made by discarding some terms. Even for linear systems, these approximations lead to energy interchange between the two scales across the interfaces. See Appendix A and [25]. Finally, our pseudo-spectral multiscale method applies directly to non-nearest neighbor interactions [19], as shown in Appendix B.

\subsection{Examples}

\subsubsection{A linear example: harmonic lattice}

To better illustrate the interfacial conditions, we consider a harmonic lattice in one space dimension with $\left(n_{a}+2\right)$ atoms. The position of the $n$th atom at rest is $x_{n}=-K+n h_{a}$ for $n=0, \ldots, n_{a}+1$, with $2 K=\left(n_{a}+1\right) h_{a}$. Its displacement is denoted by $u_{n}(t)$. With both ends fixed, we have rescaled governing equations 


$$
\ddot{u}=D_{A} u, \quad u_{0}(t)=u_{n_{a}+1}(t)=0, \quad D_{A}=\left[\begin{array}{cccc}
-2 & 1 & & \\
1 & -2 & \ddots & \\
& \ddots & \ddots & 1 \\
& & 1 & -2
\end{array}\right]_{n_{a} \times n_{a}} .
$$

Consider an $\Omega_{\mathrm{C}} / \Omega_{\mathrm{D}}$ interface at the $n_{b}$ th atom, as shown in Fig. 2. We take $L=D_{A}$. Correspondingly, the eigen-modes are

$$
\Psi_{j}=\sqrt{\frac{2}{n_{a}}}\left(\sin \left(\mathrm{j} \omega_{0}\right), \ldots, \sin \left(n_{a} \mathrm{j} \omega_{0}\right)\right)^{\mathrm{T}}, \quad \eta_{j}=-4 \sin ^{2}\left(\mathrm{j} \omega_{0} / 2\right),
$$

with $\omega_{0}=\pi /\left(n_{a}+1\right), j=1, \ldots, n_{a}$. We take the lowest $n_{\mathrm{c}}$ modes to compose the interpolation matrix $N$. The corresponding diagonal matrix is $H=\operatorname{diag}\left(\eta_{1}, \ldots, \eta_{n_{\mathrm{c}}}\right)$. have

If the initial fine fluctuations away from the MD region are negligible, we take $u_{\mathrm{C}}^{\prime}(0)=\dot{u}_{\mathrm{C}}^{\prime}(0)=0$, and

$$
\ddot{u}_{\mathrm{C}}^{\prime}=D_{\mathrm{C}} u_{\mathrm{C}}^{\prime}+\left[\begin{array}{c}
u_{n_{b}}^{\prime} \\
0 \\
\vdots \\
0
\end{array}\right], \quad D_{\mathrm{C}}=\left[\begin{array}{cccc}
-2 & 1 & & \\
1 & -2 & \ddots & \\
& \ddots & \ddots & 1 \\
& & 1 & -2
\end{array}\right] .
$$

We obtain a simple and exact formula, corresponding to (13)

$$
u_{n_{b}+1}^{\prime}=\theta * u_{n_{b}}^{\prime} .
$$

Here $\theta(t)$ is the Laplace inverse transform of the $(1,1)$ entry in $\left(s^{2} I-D_{\mathrm{C}}\right)^{-1}$. By a direct computation, we get

$$
\widehat{\theta}=S \frac{1-S^{2 N}}{1-S^{2(N+1)}} \stackrel{N \rightarrow+\infty}{\longrightarrow} S,
$$

with $S=\left[s^{2}+2-s \sqrt{s^{2}+4}\right] / 2$. We approximate

$$
\phi_{1}(t)=\theta(t) \approx \mathscr{L}^{-1}(S)=-\left(\frac{J_{1}(2 t)}{t}\right)^{\prime}=\frac{2 J_{2}(2 t)}{t} .
$$

So we end up with an interfacial condition

$$
u_{n_{b}+1} \approx \bar{u}_{n_{b}+1}+\theta(t) * u_{n_{b}}^{\prime}(t) .
$$

When $t \rightarrow+\infty$, the Bessel function is approximately $J_{n}(t) \approx \sqrt{2 / \pi} t \cos (t-n \pi / 2-\pi / 4)$. Because we use the time history treatment only on the fine fluctuations, cancellations occur in the convolution. The

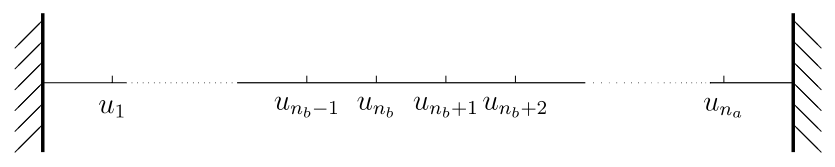

Fig. 2. Harmonic lattice in one space dimension. 


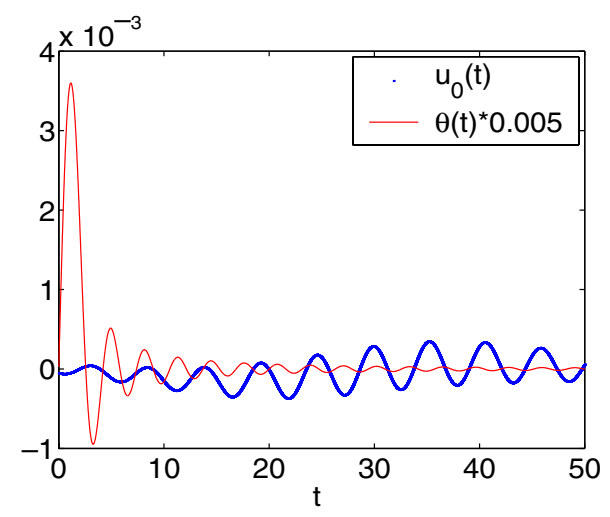

Fig. 3. The time history kernel function $\theta(t)$ and a typical time history.

function $\theta(t)$ and a typical time history is displayed in Fig. 3. Accordingly, we only keep a partial history $u_{n_{b}}^{\prime}(\tau)$ for $\tau \in(t-T, t)$ to alleviate the computing load. An upper-bound for the numerical error is

$$
\left|\int_{0}^{t-T} \theta(t-\tau) g(\tau) \mathrm{d} \tau\right| \leqslant\|g\|_{L^{2}([0, t-T])}\|\theta\|_{L^{2}([T, t])} \leqslant C\|g\|_{L^{2}([0, \infty))} T^{-1} .
$$

\subsubsection{Nonlinear examples: an anharmonic lattice and a lattice with the Lennard-Jones potential}

For a nonlinear system, we use a time history treatment corresponding to the linearized system. This is a good approximation if the nonlinear interaction between the two scales is negligible in $\Omega_{\mathrm{C}}$, namely, under the assumption of localized nonlinearity.

2.3.2.1. An anharmonic lattice. For an anharmonic lattice, we take a potential

$$
U(u)=U_{1}(u)+U_{2}(u)=\frac{1}{2} \sum_{n}\left(u_{n+1}-u_{n}\right)^{2}+\frac{K}{4} \sum_{n}\left(u_{n+1}-u_{n}\right)^{4} .
$$

Accordingly, the Newton equation is

$$
\ddot{u}_{n}=u_{n-1}-2 u_{n}+u_{n+1}+K\left[\left(u_{n+1}-u_{n}\right)^{3}-\left(u_{n}-u_{n-1}\right)^{3}\right] .
$$

Because the linearized system is precisely the harmonic lattice, we take the interfacial condition

$$
u_{n_{b}+1} \approx \bar{u}_{n_{b}+1}+\frac{2 J_{2}(2 t)}{t} * u_{n_{b}}^{\prime}(t)
$$

2.3.2.2. A lattice with the Lennard-Jones potential. The Lennard-Jones potential has been widely used for modeling atomistic interactions, e.g. $[29,18]$. In a one-dimensional lattice, the Lennard-Jones potential for a displacement vector $u=\left(u_{1}, \ldots, u_{N}\right)^{\mathrm{T}}$ is

$$
U(u)=4 \epsilon \sum_{n}\left[\left(\frac{\sigma}{r_{0}+u_{n+1}-u_{n}}\right)^{12}-\left(\frac{\sigma}{r_{0}+u_{n+1}-u_{n}}\right)^{6}\right],
$$

with $r_{0}$ being the atomic distance at rest, $\sigma$ the collision diameter and $\epsilon$ the bonding/dislocation energy. In our numerical tests, we take rescaled values of $\sigma=\epsilon=1$, and correspondingly $r_{0}=2^{1 / 6}$. 
The governing equation for displacement $u_{n}$ is

$$
\ddot{u}_{n}=-48\left[\left(r_{0}+u_{n+1}-u_{n}\right)^{-13}-\left(r_{0}+u_{n}-u_{n-1}\right)^{-13}\right]+24\left[\left(r_{0}+u_{n+1}-u_{n}\right)^{-7}-\left(r_{0}+u_{n}-u_{n-1}\right)^{-7}\right] .
$$

The linearized system is a harmonic lattice with a spring constant $k=U^{\prime \prime}\left(r_{0}\right)=72 / \sqrt[3]{2}$. The interfacial condition is

$$
u_{n_{b}+1} \approx \bar{u}_{n_{b}+1}+\frac{2 J_{2}(2 \sqrt{k} t)}{t} * u_{n_{b}}^{\prime}(t) .
$$

\subsubsection{A Slepyan model for fracture in two space dimensions}

We consider a Slepyan model describing dynamic Mode III fracture in an ideal brittle crystal [23]. For a square lattice with $n_{x} \times n_{y}$ atoms, the displacement out of the lattice plane is $u_{i j}$ at the $(i, j)$ th atom. With a damping coefficient $b \geqslant 0$, the Newton law takes the form

$$
\ddot{u}_{i j}=-b \dot{u}_{i j}+\sum_{i^{\prime}, j^{\prime}}\left(u_{i^{\prime} j^{\prime}}-u_{i j}\right) \Theta\left(2-\left|u_{i^{\prime} j^{\prime}}-u_{i j}\right|\right) \text {. }
$$

Here $\Theta(z)$ is the Heaviside step function, which describes string break when two adjacent atoms snap. An anti-plane shear is caused by external force at the upper and lower boundaries, whereas Neumann boundary conditions are applied to the left and right boundaries. These conditions are

$$
u_{i 1}=-C \sqrt{n_{y}}, \quad u_{i n_{y}}=C \sqrt{n_{y}}, \quad u_{0 j}=u_{2 j}, \quad u_{n_{x}+1, j}=u_{n_{x}-1, j} .
$$

We define a displacement vector $u_{j}=\left(u_{1 j}, \ldots, u_{n_{x} j}\right)^{\mathrm{T}}$ for the $j$ th layer of atoms. The full displacement (except for the top and bottom layers) is arranged in vector $u=\left(u_{2}, \ldots, u_{n_{y}-1}\right)^{\mathrm{T}}$.

We define the MD region $\Omega_{\mathrm{D}}$ as a strip containing layers $j=j_{m}, \ldots, j_{M}$. The crack propagates inside this region. Away from the crack, we have a damped harmonic lattice in two space dimensions. The linearized system then reads

$$
\ddot{u}_{i j}=-b \dot{u}_{i j}+\left(u_{i+1, j}-2 u_{i j}+u_{i-1, j}\right)+\left(u_{i, j-1}-2 u_{i j}+u_{i, j-1}\right),
$$

which may be written in a vector form as follows:

$$
\ddot{u}=-b \dot{u}+\left[\begin{array}{cccc}
D-2 I & I & & \\
I & D-2 I & \ddots & \\
& \ddots & \ddots & I \\
& & I & D-2 I
\end{array}\right] u, \quad D=\left[\begin{array}{ccccc}
-2 & 2 & & & \\
1 & -2 & 1 & & \\
& \ddots & \ddots & \ddots & \\
& & 1 & -2 & 1 \\
& & & 2 & -2
\end{array}\right] .
$$

2.3.3.1. Decomposition of the displacements. The spectral decomposition is performed in the following way. We first subtract a linear stretching part from the displacement:

$$
w_{i j}=u_{i j}-\left[\frac{2(j-1)}{n_{y}-1}-1\right] C \sqrt{n_{y}} .
$$

The unstretched displacement $w$ satisfies the same linear Newton law (30), but with a homogeneous Dirichlet boundary condition $w_{i 1}=w_{i n_{y}}=0$. The $(l, m)$ th mode has an eigenvalue $\eta_{l m}=2\left(\cos \left[(l-1) \omega_{x}\right]\right.$ $\left.-1)+2\left(\cos (m-1) \omega_{y}\right]-1\right)\left(l=1, \ldots, n_{x}, m=2, \ldots, n_{y}-1\right)$, and an eigenvector

$$
\Psi^{l m}=\left[\begin{array}{c}
\Psi_{1}^{l m} \\
\vdots \\
\Psi_{n_{y}}^{l m}
\end{array}\right], \quad \Psi_{k}^{l m}=\sin \left[(k-1)(m-1) \omega_{y}\right]\left[\begin{array}{c}
\cos \left[(l-1) \omega_{x}\right] \\
\vdots \\
\cos \left[\left(n_{x}-1\right)(l-1) \omega_{x}\right]
\end{array}\right]
$$

with frequencies $\omega_{x}=\pi /\left(n_{x}-1\right), \omega_{y}=\pi /\left(n_{y}-1\right)$. 
We expand $w$ in these normal modes, and pick the lowest $N_{x} \times\left(N_{y}-2\right)$ modes $\left(l=1, \ldots, N_{x}, m=2, \ldots\right.$, $\left.N_{y}-1\right)$, with $N_{x}=\left(n_{x}-1\right) / p_{x}+1, N_{y}=\left(n_{y}-1\right) / p_{y}+1$. We define an interpolation matrix

$$
N=\left(N_{2}, \ldots, N_{N_{y}-1}\right) \quad \text { with } N_{J}=\left(\Psi^{1 J}, \ldots, \Psi^{N_{x} J}\right) .
$$

The governing equation for the the fine fluctuation $w^{\prime}=\left(I-N N^{\mathrm{T}}\right) w$ is

$$
\ddot{w}^{\prime}=-b \dot{w}^{\prime}+\left[\begin{array}{cccc}
D-2 I & I & & \\
I & D-2 I & \ddots & \\
& \ddots & \ddots & I \\
& & I & D-2 I
\end{array}\right] w^{\prime} .
$$

2.3.3.2. The interfacial conditions. Now we derive an interfacial condition for the upper interface, i.e. the $j_{M}$ th layer. In computing the interfacial conditions, we should modify the formula (13) by including the effects of the damping term. It may be shown that $\Phi_{\mathrm{G}}$ is the upper-left block of $\mathscr{L}^{-1}\left(s^{2} I+s b I-L_{\mathrm{CC}}\right)$ with

$$
L_{\mathrm{CC}}=\left[\begin{array}{cccc}
D-2 I & I & & \\
I & D-2 I & \ddots & \\
& \ddots & \ddots & I \\
& & I & D-2 I
\end{array}\right] .
$$

Taking a matrix $R$ whose $(l, m)$ th entry is $\cos [(l-1)(m-1) \omega x]$, we diagonalize $L_{\mathrm{CC}}$ by a transform matrix $\operatorname{diag}(R, \ldots, R)$. It is easy to check that $R^{-1} D R=\operatorname{diag}\left(\zeta_{1}, \ldots, \zeta_{n_{x}}\right) \equiv H_{1}$ for $\zeta_{k}=2\left(\cos \left[2(i-1) \omega_{x}\right]-1\right)$. In the limit of a semi-infinite sheet, we may compute $w_{j_{M}+1}^{\prime}$ from

$$
w_{j_{M}+1}^{\prime}=R \operatorname{diag}\left(\theta_{1}, \ldots, \theta_{n_{x}}\right) * R^{-1} w_{j_{M}}^{\prime} .
$$

Here

$$
\hat{\theta}_{i}=\frac{1}{2}\left[\left(s+\frac{b}{2}\right)^{2}+2 c_{i}-\sqrt{\left(\left(s+\frac{b}{2}\right)^{2}+2 c_{i}\right)^{2}-4}\right],
$$

with $c_{i}=2-\zeta_{i}-b^{2} / 4$. The kernel functions $\theta_{i}(t)$ may be computed numerically (see Appendix C).

By virtue of the special form of $R$, we use FFT for the transform, which is very efficient. The original variable $u_{j_{M}+1}$ is recovered from

$$
u_{j_{M}+1}=\bar{w}_{j_{M}+1}+w_{j_{M}+1}^{\prime}+\left(\frac{2 j_{M}}{n_{y}-1}-1\right) C \sqrt{n_{y}} .
$$

Here the mean displacement $\bar{w}_{j_{M}+1}$ is computed with $\tilde{d}$ by an equation similar to (69), with a modification due to the damping term.

The same treatment applies to the lower layer $j=j_{m}$.

\section{A pseudo-spectral approximation and coarse grid equations}

\subsection{A pseudo-spectral approximation}

In the previous discussions, we have derived the governing equation (7) for the low order mode amplitude $\tilde{d}$. As mentioned before, this is not a closed system. Moreover, because a component in $\tilde{d}$ is a mode 
amplitude, it involves displacement over the whole domain. Thus we face a situation similar to solving partial differential equations by a spectral method. For nonlinear problems, it is known that a pseudo-spectral method can handle nonlinear terms more efficiently.

We first define a coarse grid over the whole domain $\Omega$. At each coarse grid point $y_{J}$, we assign a displacement $d_{J}$. These displacements form a coarse grid displacement vector $d$. Note that both $d$ and $\tilde{d}$ describe the long wave length components of the total displacement $u$, and we may approximate $\tilde{d}$ (and hence the mean displacement $\bar{u}$ ) with the information of $d$. The dynamics of $d$ are local and relatively easier to compute. In the following, we propose a matching differential operator method to derive governing equations for $d$. This method allows us to reach any desired order of accuracy, up to the spectral resolution of the selected coarse grid.

\subsection{A motivating example: harmonic lattice}

The right-hand side of the Newton equation (14) approximates the second-order derivative to the leading order.

$$
u_{t t}=h_{a}^{2} u_{x x}+\mathrm{O}\left(h_{a}^{4}\right)
$$

For the MC computation, we take coarse grid points located at $y_{J}=-L+J h_{e}$ for $J=0, \ldots, n_{\mathrm{c}}+1$, where $h_{e}=p h_{a}$ and $n_{a}+1=p\left(n_{c}+1\right)$. It is then natural to design an equation for the coarse grid displacement $d$ as follows:

$$
\ddot{d}_{J}=\frac{1}{p^{2}}\left(d_{J-1}-2 d_{J}+d_{J+1}\right) .
$$

We may regard the full MD system (14) and the coarse grid equations (41) as two numerical approximations to the same wave equation, yet with different grid sizes. After interpolations, these two numerical solutions are expected to differ only by the order of $\mathrm{O}\left(h_{a}^{4}\right)$. In principle, we can design a governing equation for $d$, which has the same underlying partial differential equation as the Newton law to a higher order in $h_{a}$. This is the basic idea of the matching differential operator method.

For an infinitely long harmonic lattice, we use the Taylor series to obtain

$$
\ddot{u}=u\left(x-h_{a}, t\right)-2 u(x, t)+u\left(x+h_{a}, t\right)=\sum_{m=1}^{\infty} \frac{2}{(2 m) !} \frac{\partial^{2 m} u}{\partial x^{2 m}} h_{a}^{2 m} \equiv D_{A} u .
$$

We define a central difference operator $D$ by $D d=d\left(y-p h_{a}, t\right)-2 d(y, t)+d\left(y+p h_{a}, t\right)$. It corresponds to a central difference in the coarse grid. We expand it as

$$
D d=\sum_{m=1}^{\infty} \frac{2}{(2 m) !} \frac{\partial^{2 m} d}{\partial y^{2 m}}\left(p h_{a}\right)^{2 m}
$$

We take an operator set $\mathscr{K}=\left\{D, D^{2}, \ldots\right\}$. For appropriate coefficients $B_{k} \in \mathbb{R}$ to be determined, we construct the equation

$$
\ddot{d}=\sum_{k=1}^{\infty} B_{k} D^{k} d
$$

to approximate (42).

We may solve $B_{k}$ by equating coefficients of $h_{a}^{l}$ in (42) and the expansion of (44)

$$
\sum_{k=1}^{\infty} \frac{2 h_{a}^{2 k}}{(2 k) !} \frac{\partial^{2 k}}{\partial x^{2 k}}=\sum_{k=1}^{\infty} B_{k}\left[\sum_{m=1}^{\infty} \frac{2\left(p h_{a}\right)^{2 m}}{(2 m) !} \frac{\partial^{2 m}}{\partial x^{2 m}}\right]^{k} .
$$


This fixes the coarse grid equation. For example, with an error to the order of $\mathrm{O}\left(h_{a}^{10}\right)$, a scheme can be designed as

$$
\ddot{d}=\frac{D}{p^{2}}\left\{1-\frac{\left(p^{2}-1\right)}{12 p^{2}} D\left[1-\frac{4 p^{2}-1}{30 p^{2}} D\left(1-\frac{9 p^{2}-1}{56 p^{2}} D\right)\right]\right\} d .
$$

Lower order schemes are obtained by dropping high order terms in $D$. Meanwhile, for a finite lattice, there is a limited number of coarse grid points, leading to a limitation in the order of accuracy that can be reached. If we choose the operator set properly, we may reproduce a spectral method for the coarse grid. That is, the limit in accuracy is a spectral resolution of the selected coarse grid.

The harmonic lattice is linear, and we remark that the matching differential operator approach has an alternative derivation by matching the dispersion relation. Moreover, it is easy to find that the Newton equation takes a dispersion relation

$$
\lambda^{2}=2(1-\cos \omega)
$$

With $z=2 \sin (\omega p / 2)$, the scheme (46) takes a dispersion relation

$$
\mu^{2}=\frac{z^{2}}{p^{2}}\left\{1+\frac{p^{2}-1}{12 p^{2}} z^{2}\left[1+\frac{4 p^{2}-1}{30 p^{2}} z^{2}\left(1+\frac{9 p^{2}-1}{56 p^{2}} z^{2}\right)\right]\right\} .
$$

Here $\mu$ approximates $\lambda$, corresponding to the approximation in (46) for the original Newton law (14).

\subsection{A matching differential operator method}

For the sake of clarity, we adopt a conventional notation of multi-index, e.g. $n=\left(n_{x}, n_{y}, n_{z}\right)$.

We imagine a continuous displacement 'field' $u(x, t)$ in the Lagrangian coordinate, and study its evolution. The displacement of the $n$th atom is recovered from $u_{n}(t)=u\left(x_{n}, t\right)$, with $x_{n}$ its position at rest. We take the same notation $u$ for the field and the displacement vector, when there is no confusion. Furthermore, the atomic position has been rescaled by a characteristic macroscopic scale. This means that the characteristic atom distance $h_{a}$ is a small quantity, and the distance between two adjacent atoms $h_{i}=\left|x_{n+i}-x_{n}\right|=\mathrm{O}\left(h_{a}\right)$.

The dynamics for $u_{n}$ are governed by the potential gradient at this atom. We present the method for nonnearest neighbor interactions here. Without loss of generality, we assume an internal force depending on adjacent atoms $u_{n+i}$ for $|i| \leqslant I$. It reduces to nearest-neighbor interactions if $I=1$.

We further imagine a continuous density field $m(x)$ and external force field $f_{\text {ext }}(x, t)$. The Newton law for this atom can be put as

$$
m\left(x_{n}\right) \frac{\partial^{2} u\left(x_{n}, t\right)}{\partial t^{2}}=f\left(u\left(x_{n+i}, t\right)_{|i| \leqslant I}\right)+f_{\text {ext }}\left(x_{n}, t\right) .
$$

We make the Taylor expansion for an adjacent atom with

$$
u\left(x_{n+i}, t\right)=u\left(x_{n}, t\right)+\sum_{s=1}^{\infty} \frac{1}{s !} \frac{\partial^{s} u\left(x_{n}, t\right)}{\partial x^{s}} h_{i}^{s} .
$$

Plugging into the previous equation, we obtain an underlying differential equation around this atom (coordinate $\left(x_{n}, t\right)$ suppressed)

$$
\begin{aligned}
\frac{\partial^{2} u}{\partial t^{2}} & =\frac{1}{m}\left[f\left(u+\sum_{s=1}^{\infty} \frac{1}{s !} \frac{\partial^{s} u}{\partial x^{s}} h_{i}^{s}\right)_{|i| \leqslant I}+f_{\mathrm{ext}}\right] \\
& \equiv F\left(u, u_{x}, u_{x x}, \ldots ; x, t\right)
\end{aligned}
$$


If $f$ is a multivariate function with enough regularity, we may cut-off the internal force expression to $\mathrm{O}\left(h_{a}^{l+1}\right)$ for any $l \in \mathbb{N}$. This leads to a closed form of the approximated internal force, involving $u$ and its finite spatial derivatives. Formally, we write it as

$$
\frac{\partial^{2} u}{\partial t^{2}}=F_{l}\left(u, u_{x}, \ldots, \frac{\partial^{l} u}{\partial x^{l}}\right)+\mathrm{O}\left(h_{a}^{l+1}\right) .
$$

To get a coarse grid equation, we further assume a reduced displacement vector $d$ of size smaller than $u$. We imagine each component $d_{J}$ representing a displacement of a 'virtual particle cluster' at position $y_{J}$ with unitary mass. Though a cluster is bigger than an atom, we assume that it occupies a volume much smaller than the the macroscopic scale. Accordingly, there is a characteristic cluster distance $h_{e} \ll 1$, for which we have $\left|y_{J+1}-y_{J}\right|=\mathrm{O}\left(h_{e}\right)$. This allows us to consider another 'displacement field' $d(x)$ for which $d_{J}=d\left(y_{J}\right)$.

We select a set of differential operators $\mathscr{K}=\left\{K_{\alpha}\right\}$. Each operator is chosen to be related to a few adjacent atoms, numerically easy to compute, and not explicitly relying on $y_{J}$. These operators are in general nonlinear. Though the choice of the operator set is usually not as obvious as for the harmonic lattice, mathematical and physical considerations can be used for a suitable selection. We shall demonstrate the choice of $\mathscr{K}$ by some examples in Section 3.4.

An operator is of $l$ th order, if its Taylor expansion leads to a residual of $(l+1)$ st order. For instance, the central difference operator $D_{\mathrm{c}}$ defined by $\left(D_{\mathrm{c}} d\right)_{n}=d_{n+1}-d_{n-1}$ is of second order, whereas the forward difference $D_{+}$defined by $\left(D_{+} d\right)_{n}=d_{n+1}-d_{n}$ is of first order.

We collect a subset of the same order $l$ as $\mathscr{K}_{l}=\left\{K_{l, 1}, \ldots, K_{l, n(l)}\right\}$, and $\mathscr{K}=\bigcup_{l} \mathscr{K}_{l}$.

With parameters $B_{i j}$ to be determined, we construct a coarse grid equation for $d$

$$
\ddot{d}=\sum_{i=1}^{l} \sum_{j=1}^{n(i)} B_{i j} K_{i, j} d+g_{\text {ext }} .
$$

In the same way, we make approximations using Taylor expansions. Formally, we write an approximate equation with parameters $B_{i j}$ as

$$
\frac{\partial^{2} d}{\partial t^{2}}=G_{l}\left(d, d_{x}, d_{x x}, \ldots, \frac{\partial^{l} d}{\partial x^{l}} ; x, B_{i j}\right)+\mathrm{O}\left(h_{e}^{l+1}\right) .
$$

Comparing (54) with (52), we may find $B_{i j}$ to match the Taylor expansion coefficients to the desired order. This determines the coarse grid equation. It solves the same underlying partial differential equation as the Newton law, up to a desired order. It is conceivable that $d(x) \approx u(x)$ to the same order. For various numerical examples in this paper, typically the leading term in the Newton equation is of the order of $\mathrm{O}\left(h_{a}^{2}\right)$. In (54), we match up to the order $h_{a}^{l+1}$. We subtract 2 from the order, and call it an MDO$(k-1)$ scheme for brevity. The corresponding multiscale method is referred to as a pseudo-spectral MDO- $(k-1)$ scheme.

We remark that for multiscale computations, the coarse grid equations may be extracted by either a discrete or a continuous approach. Many multiscale methods take a discrete approach, where one assumes a reduced displacement vector, and derives a governing equation by making some form of approximations $[4,21,22,24,28]$.

In contrast, in a continuous approach, one assumes a continuous 'displacement field' instead. Equations derived in this manner typically bear nice features such as simplicity, clarity and locality. The equation of continuum may be regarded as a special case.

The matching differential operator method adopts a continuous approach, sharing some similar ideas with [6]. It is a systematic way for deriving coarse grid equations. We can also construct higher order 
coarse grid equations. Same as for the harmonic lattice, in the limit of including all coarse grid points, we may choose a suitable operator set $\mathscr{K}$, and derive an equation on $d$ that is equivalent to a spectral method for the coarse grid approximation of the Newton laws. Therefore, the matching differential approach provides a way to reach arbitrary desired order of accuracy, up to a spectral resolution of a selected coarse grid.

\subsection{Examples}

\subsubsection{Nonlinear examples in one space dimension}

3.4.1.1. Anharmonic lattice. The linear part of the interatomic force in (23) is related to the same operator as that for the harmonic lattice

$$
D_{A}=\sum_{m=1}^{\infty} \frac{2}{(2 m) !} \Delta^{m}
$$

For the nonlinear force, we expand

$$
\left(u_{n+1}-u_{n}\right)^{3}-\left(u_{n}-u_{n-1}\right)^{3}=2 \sum_{k=1}^{\infty}\left(h_{a}\right)^{2(k+1)} \mathscr{L}_{k}(u),
$$

where the operator $\mathscr{L}_{k}$ is given by

$$
\mathscr{L}_{k-1}(f)=\sum_{3 k_{1}=2 k}\left(\frac{f^{\left(k_{1}\right)}}{k_{1} !}\right)^{3}+3 \sum_{\substack{2 k_{1}+k_{2}=2 k \\ k_{1} \neq k_{2}}} \frac{\left(f^{\left(k_{1}\right)}\right)^{2} f^{\left(k_{2}\right)}}{\left(k_{1} !\right)^{2} k_{2} !}+6 \sum_{\substack{1 \leqslant k_{1}<k_{2}<k_{3} \\ k_{1}+k_{2}+k_{3}=2 k}} \frac{f^{\left(k_{1}\right)} f^{\left(k_{2}\right)} f^{\left(k_{3}\right)}}{k_{1} ! k_{2} ! k_{3} !} .
$$

We assume that the coefficient for nonlinearity is of the order $h_{a}^{-2}$, i.e. $K=\bar{K} / h_{a}^{2}$. Adding the previous terms together, we have

$$
\ddot{u}_{n}=2 \sum_{k=1}^{\infty} h_{a}^{2 k}\left[\frac{u^{(2 k)}}{(2 k) !}+\bar{K} \mathscr{L}_{k}(u)\right] .
$$

Similar computations yield

$$
\left(d_{I+1}-d_{I}\right)^{3}-\left(d_{I}-d_{I-1}\right)^{3}=2 \sum_{k=1}^{\infty}\left(p h_{a}\right)^{2(k+1)} \mathscr{L}_{k}(d) .
$$

Noticing that

$$
\mathscr{L}_{2}(d)=\left(\frac{d_{x x}}{2}\right)^{3}+\frac{d_{x}^{2} d_{x x x x}}{8}+\frac{d_{x} d_{x x} d_{x x x}}{2}=\frac{1}{8}\left(d_{x} d_{x x}^{2}+d_{x}^{2} d_{x x x}\right)_{x},
$$

we may design an MDO-4 scheme as follows:

$$
\begin{aligned}
\ddot{d}_{I}= & \frac{1}{p^{2}}(D d)_{I}-\frac{p^{2}-1}{12 p^{4}}\left(D^{2} d\right)_{I}+\frac{\bar{K}}{h_{a}^{2} p^{4}}\left[\left(D_{+} d\right)_{I}^{3}-\left(D_{-} d\right)_{I}^{3}\right]-\frac{\bar{K}\left(p^{2}-1\right)}{4 h_{a}^{2} p^{6}}\left[(D d)_{I}^{3}+\left(D_{\mathrm{c}} d\right)_{I}^{2}\left(D^{2} d\right)_{I}\right. \\
& \left.+4\left(D_{\mathrm{c}} d\right)_{I}(D d)_{I}\left(D_{\mathrm{c}} D d\right)_{I}\right] .
\end{aligned}
$$

The subscript $I$ denotes the $I$ th component of the vector, and $\left(D_{+} d\right)^{3}$ means componentwise cubic power of the vector $D_{+} d$.

Though not explicitly written, here the differential operator set $\mathscr{K}$ includes not only linear operators $D^{k}$, but also nonlinear operators involved terms like $\left(D_{+} d\right)^{3}$. 
3.4.1.2. A lattice with the Lennard-Jones potential. Similar to the anharmonic lattice, an MDO-4 scheme for (26) is designed as

$$
\begin{aligned}
\ddot{d}_{I}= & -\frac{48}{p}\left[\left(r_{0}+\frac{d_{I+1}-d_{I}}{p}\right)^{-13}-\left(r_{0}+\frac{d_{I}-d_{I-1}}{p}\right)^{-13}\right]+\frac{24}{p}\left[\left(r_{0}+\frac{d_{I+1}-d_{I}}{p}\right)^{-7}-\left(r_{0}+\frac{d_{I}-d_{I-1}}{p}\right)^{-7}\right] \\
& +\frac{\left(p^{2}-1\right)\left(-52 r_{0}^{-14}+14 r_{0}^{-8}\right)}{p^{4}}\left(d_{I-2}-4 d_{I-1}+6 d_{I}-4 d_{I+1}+d_{I+2}\right) .
\end{aligned}
$$

\subsubsection{The Slepyan model for fracture in two space dimensions}

Because the system (28) is a damped linear harmonic lattice in each dimension, we treat it in the same way as in the one-dimensional lattice. The coarse grid MDO-4 equation is

$$
\begin{aligned}
\ddot{d}_{I J}= & -b \dot{d}_{I J}+\frac{1}{p_{x}^{2}}\left(d_{I-1, J}-2 d_{I J}+d_{I+1, J}\right)+\frac{1}{p_{y}^{2}}\left(d_{I, J-1}-2 d_{I J}+d_{I, J+1}\right) \\
& -\frac{p_{x}^{2}-1}{12 p_{x}^{4}}\left(d_{I-2, J}-4 d_{I-1, J}+6 d_{I J}-4 d_{I+1, J}+d_{I+2, J}\right)-\frac{p_{y}^{2}-1}{12 p_{y}^{4}}\left(d_{I, J-2}-4 d_{I, J-2}+6 d_{I J}-4 d_{I, J+1}+d_{I, J+2}\right) .
\end{aligned}
$$

\section{Numerical implementation}

\subsection{Time integration}

For time integration of both the MD computation and the coarse grid computation, we use a verlet algorithm. We illustrate this algorithm for

$$
\ddot{q}=A(q, t) \text {. }
$$

With a time step size $\Delta t$ and data $q\left(t^{n}\right)=q^{n}, \dot{q}\left(t^{n}\right)=\dot{q}^{n}$, the acceleration is computed as $A^{n}=A\left(q^{n}, t^{n}\right)$. We update one step for $q$ and one half step for $\dot{q}$ in

$$
q^{n+1}=q^{n}+\dot{q}^{n} \Delta t+A^{n} \frac{(\Delta t)^{2}}{2}, \quad \dot{q}^{n+1 / 2}=\dot{q}^{n}+A^{n} \frac{\Delta t}{2} .
$$

A new acceleration $A^{n+1}=A\left(q^{n+1}, t^{n+1}\right)$ is computed, and we update another half step for $\dot{q}$ by

$$
\dot{q}^{n+1}=\dot{q}^{n+1 / 2}+A^{n+1} \frac{\Delta t}{2} .
$$

This second-order scheme is applied to the dynamics of both $d$ and $u_{\mathrm{D}}$. Furthermore, we use a mixed time integration technique. That is, we take a time step size $\Delta \tau$ for computing $u_{\mathrm{D}}$, and $\Delta t=m \Delta \tau$ for computing $d$.

\subsection{A flowchart of the pseudo-spectral multiscale method}

We summarize the pseudo-spectral multiscale method in the following list, with a mesh schematically depicted in Fig. 4. 


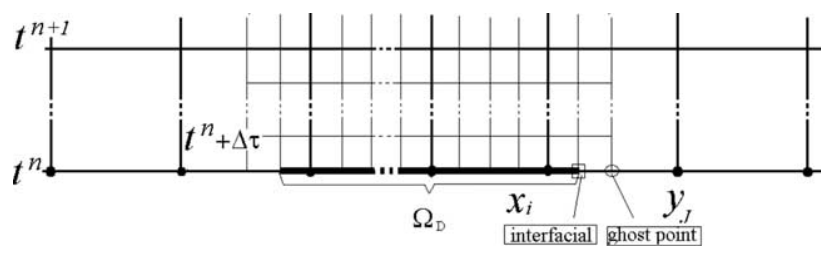

Fig. 4. Illustration of computing mesh.

(1) The coarse grid displacement $d$ is updated in $\Omega$ with a time step size $\Delta t$ by solving the coarse grid equation:

$\ddot{d}=\sum_{\alpha \in \mathscr{I}} b_{\alpha} D_{\alpha} d+g_{\text {ext }}$.

The precise form of the equation is devised by the matching differential operator approach.

(2) Holding the velocity for $d$ fixed at $t^{n}$, we compute the corresponding spectral representation from

$\tilde{d} \approx R^{-1} d, \quad \dot{\tilde{d}} \approx R^{-1} \dot{d}, \quad R=\left(\widetilde{\Psi}_{1}, \ldots, \widetilde{\Psi}_{3 n_{\mathrm{c}}}\right)$.

At sub-time steps $t^{n}+k \Delta \tau$ (for $k=1, \ldots, m$ ), we compute the mean displacement $\bar{u}=N \tilde{d}$ at the interfacial atoms and ghost point atoms with

$\tilde{d}_{J}\left(t^{n}+k \Delta \tau\right)=\tilde{d}_{J}\left(t^{n}\right) \cos \left(\sqrt{-\eta_{J}} k \Delta \tau\right)+\frac{\dot{\tilde{d}}_{J}\left(t^{n}\right)}{\sqrt{-\eta_{J}}} \sin \left(\sqrt{-\eta_{J}} k \Delta \tau\right)+f_{\mathrm{ext}} \frac{(k \Delta \tau)^{2}}{2}$.

(3) Within each sub-time step, we compute the fine fluctuation at the ghost point atoms $u_{\mathrm{G}}^{\prime}$ with a subgrid time step size $\Delta \tau$ by solving

$u_{\mathrm{G}}^{\prime} \approx \widetilde{\Phi}_{\mathrm{G}}(t) *\left[M_{A_{\mathrm{C}}}^{-1}\left(L_{\mathrm{CD}} u_{\mathrm{D}}^{\prime}+\widetilde{Q}_{\mathrm{C}}^{\mathrm{T}} f_{\mathrm{ext}}\right)\right]+\dot{\widetilde{\Phi}}_{\mathrm{G}}(t) u_{\mathrm{C}}^{\prime}(0)+\widetilde{\Phi}_{\mathrm{G}}(t) \dot{u}_{\mathrm{C}}^{\prime}(0)$.

Here $\Phi_{\mathrm{G}}(t)$ is a submatrix of

$\Phi=\mathscr{L}^{-1}\left[\left(s^{2} I-M_{A_{\mathrm{C}}}^{-1} L_{\mathrm{CC}}\right)^{-1}\right]$.

In $\Omega_{\mathrm{D}}$, we compute a subsystem of the Newton law

$M_{A_{\mathrm{D}}} \ddot{u}_{\mathrm{D}}=f_{\mathrm{D}}+f_{\text {ext,D}}$

with the interfacial conditions of ghost point atom displacement $u_{\mathrm{G}}=\bar{u}_{\mathrm{G}}+u_{\mathrm{G}}^{\prime}$. We update the time history of $M_{A_{\mathrm{C}}}^{-1} L_{\mathrm{CD}} u_{\mathrm{D}}^{\prime}$ after each sub-time step.

(4) After finishing $m$ steps of MD computations, we have $u_{\mathrm{D}}$ at time $t^{n}+m \Delta \tau=t^{n}+\Delta t$. The average of $u_{\mathrm{D}}$ typically gives $d_{\mathrm{D}}$ different from that by MC computation. We force consistency by reassignment of $d$ and $\dot{d}$. A simple choice is to take the mean value of the MD solution for nearby $2 p+1$ atoms around a coarse grid point.

In step (4), we make use of the MD solution to enforce consistency with the MC computation. In the next time step, we use the new value to update $d$. Information is then naturally passed from the MD region $\Omega_{\mathrm{D}}$ to the MC region $\Omega_{\mathrm{C}}$. Moreover, by this procedure we avoid using a handshaking region. This is another feature of the pseudo-spectral multiscale method which differs from most existing multiscale methods. 


\subsection{Computational costs}

To estimate the computing load, we consider a typical case with $n_{a}$ atoms in the lattice. For our multiscale computations, we take $n_{\mathrm{c}}$ coarse grid points, $n_{\mathrm{D}}$ atoms in MD region, $n_{\mathrm{G}}$ ghost point atoms, and keep a time history of period $T=n_{\mathrm{H}} \Delta \tau$. The number of interfacial atoms is on the same order as the ghost point atoms.

In one big time step $\Delta t$, the coarse grid update creates a computing load on the order of $\mathrm{O}\left(n_{\mathrm{c}}\right)$. The spectral decomposition by FFT technique requires a computing load $\mathrm{O}\left(n_{\mathrm{c}} \log n_{\mathrm{c}}\right)$. In each time step $\Delta \tau$, the MD update mainly includes the following computations. Finding the mean displacement at ghost point atoms and interfacial atoms requires $\mathrm{O}\left(n_{\mathrm{G}} \log n_{\mathrm{G}}\right)$ computations. The time history convolution requires $\mathrm{O}\left(n_{\mathrm{G}} n_{\mathrm{H}}\right)$ computations. The update of the MD displacement, velocity and acceleration requires $\mathrm{O}\left(n_{\mathrm{D}}\right)$ computations. Other computations are of the order $\mathrm{O}\left(n_{\mathrm{D}}\right)$. Summing them together, we find the total computing load to be $\mathrm{O}\left(n_{\mathrm{c}} \log n_{\mathrm{c}}+m\left(n_{\mathrm{G}}\left(n_{\mathrm{H}}+\log n_{\mathrm{G}}\right)+n_{\mathrm{D}}\right)\right)$.

As a comparison, the update of one $\Delta t$ step takes $\mathrm{O}\left(m n_{a}\right)$ operations for a full MD computation with a step size $\Delta \tau$. On the other hand, in a multiscale method such as the BSM, the computing load is $\mathrm{O}\left(n_{\mathrm{c}}+m\left(n_{\mathrm{G}} n_{\mathrm{H}}+n_{\mathrm{D}}\right)\right)$. The pseudo-spectral multiscale method has slightly more computations since it uses FFT. The gain in resolution is worth this additional cost.

\section{Numerical results}

We present our numerical results to demonstrate nice features of the pseudo-spectral multiscale method. We perform simulations for the harmonic lattice, anharmonic lattice, and the lattice with the LennardJones potential, as well as the Slepyan model for fracture in two space dimensions. For comparisons, we make also computations by full MD simulations. We shall refer to full MD solutions as exact solutions. Meanwhile, for multiscale computations, we use MD to represent a solution by MD computation in $\Omega_{\mathrm{D}}$ and $\mathrm{MC}$ to represent that by coarse grid computation in $\Omega_{\mathrm{C}}$. We remark that these numerical examples have a much smaller size than the real applications.

\subsection{Harmonic lattice}

A harmonic lattice is the simplest model for molecular dynamics of a crystal, and serves as a test problem for our multiscale methods.

In our numerical simulations, we take $h_{a}=0.005, p=10$ and $h_{e}=p h_{a}=0.05$. The initial condition is given by $u_{n}(0)=u^{0}\left(x_{n}\right)$ with

$$
u^{0}(x)= \begin{cases}0.005 \frac{\mathrm{e}^{-100 x^{2}}-\mathrm{e}^{-6.25}}{1-\mathrm{e}^{-6.25}}(1+0.1 \cos (80 \pi x)) & \text { for }|x| \leqslant 0.25 \\ 0, & \text { elsewhere }\end{cases}
$$

First, we compare different coarse grid schemes, by performing numerical tests in the domain $x \in[-2,2]$ without the time history kernel. Solutions at time $t=150$ are depicted in Fig. 5. Because of finite propagation speed, the lattice is essentially at equilibrium far away. Furthermore, the system has a symmetry $x \rightarrow-x$, which is preserved by our numerical schemes. Therefore, we only plot solutions in part of the computing domain.

The exact solution is computed by a full MD simulation with $\Delta \tau=0.005$. In Fig. 5, we observe that the primary wave (solid line) is centered around $x=h_{a} t=0.75$, and the fine fluctuation propagates slower.

Coarse grid schemes are tested for $\Delta t=0.05$. Fine fluctuations could not be reproduced in such coarse grid. The MDO-2 scheme produces a slower phase speed and a kink at the wave tail. The MDO-4 scheme 


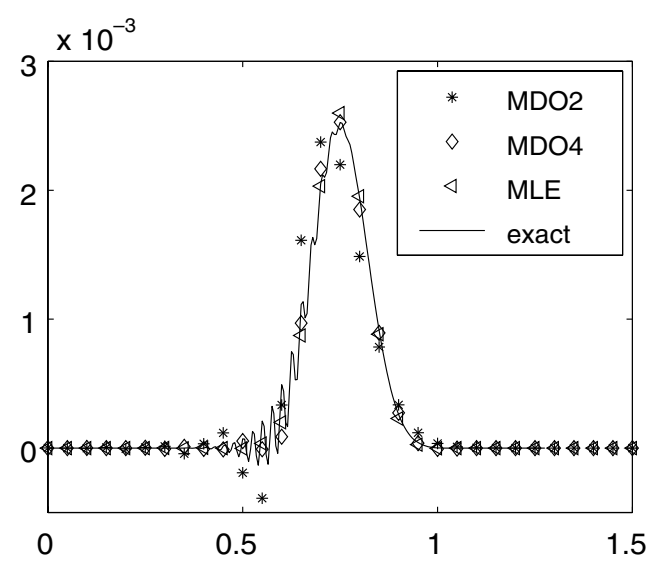

Fig. 5. Harmonic lattice $u(x, 150)$ : by the MDO-2, MDO-4 and MLE schemes.

resolves the mean displacement very well. These are clearly explained by the dispersion relations, as shown in Fig. 6.

For comparison, we use a BSM-MLE scheme. The scheme is described in Appendix A. For more details, please refer to [25,28]. We observe that the MLE scheme resolves the coarse grid very well. However, one has to perform the inverse for the square root of the effective mass matrix, which is numerically quite expensive.

Now we compute with the pseudo-spectral multiscale method. The time history is kept for $T=5$. There are 151 atoms used in $\Omega_{\mathrm{D}}=[-0.375,0.375]$.

The solution at various times is shown in Fig. 7, computed by the pseudo-spectral MDO-4 scheme. Throughout the whole process, the mean displacement in $\Omega_{\mathrm{C}}$ is faithfully reproduced, and almost no reflection is observed across the interface.

For comparison, we show results by the BSM-MLE scheme with the same numerical setting in Fig. 8. Besides long wave reflection across the interface, the mean displacement at the peak is also slightly higher.

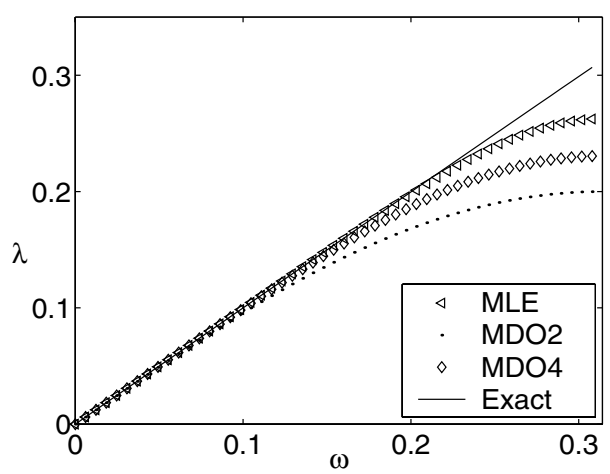

Fig. 6. Dispersion relation for the harmonic lattice by different schemes: the round frequency in time $\lambda$ is plotted versus the wave number $\omega \in[0, \pi / 10]$. 

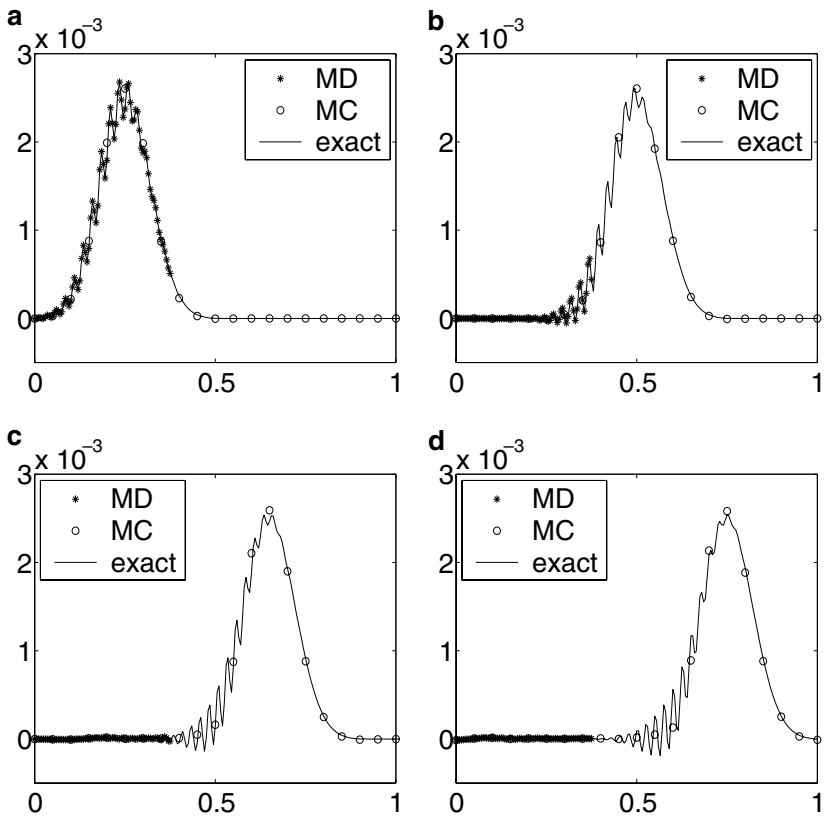

Fig. 7. Harmonic lattice by the pseudo-spectral MDO-4 method: (a) $u(x, 50)$; (b) $u(x, 100)$; (c) $u(x, 130)$ and (d) $u(x, 150)$.

See subplots (c) and (d). Moreover, as analyzed in [25], the numerical error comes mainly from energy interchange between the mean displacement and fine fluctuations. As described in Appendix A, an approximation has been made in BSM for interfacial conditions. A consequence of this approximation is a sensitivity
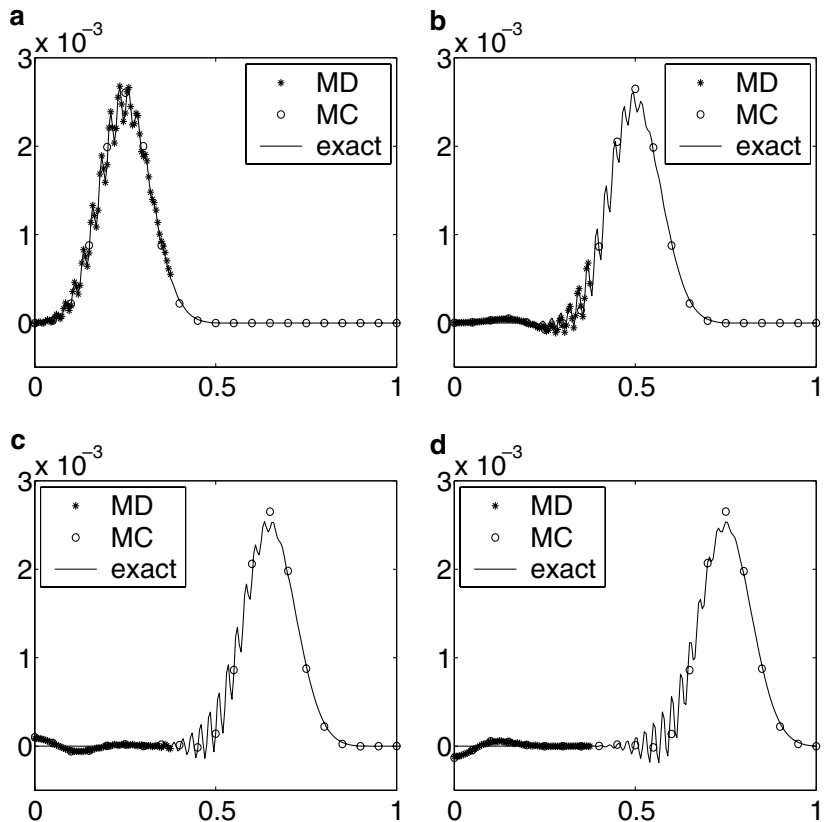

Fig. 8. Harmonic lattice by the BSM-MLE scheme: (a) $u(x, 50)$; (b) $u(x, 100)$; (c) $u(x, 130)$; and (d) $u(x, 150)$. 
to the choice of $\Omega_{\mathrm{D}}$. For instance, if we take an enlarged MD-region containing 161 atoms instead, larger reflections occur in the BSM-MLE scheme. In contrast, the pseudo-spectral MDO-4 method is not affected (see Fig. 9).

To investigate the source of error further, we make use of linearity, and separately compute the evolution with smooth initial data (mean part) and that with purely fluctuation initial data. From Fig. 10(a) and (b), we observe that the residual in the MD region is mainly due to the smooth initial data for the BSM-MLE scheme. By the pseudo-spectral multiscale method, the reflection amplitude is reduced to about $1 / 10$ of that by BSM. Moreover, we observe that the numerical error with the smooth initial data is comparable to that
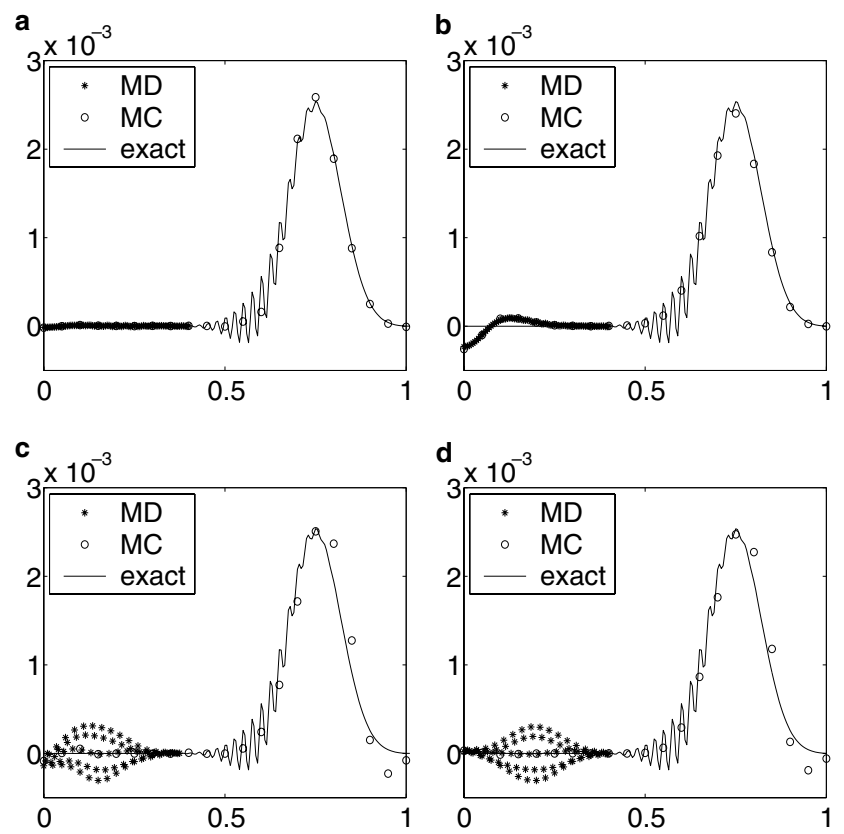

Fig. 9. Harmonic lattice $u(x, 150)$ : (a) pseudo-spectral MDO-4 scheme with enlarged $\Omega_{\mathrm{D}}$; (b) BSM-MLE scheme with enlarged $\Omega_{\mathrm{D}}$; (c) BSM scheme, no time history treatment and (d) BSM scheme with enlarged $\Omega_{\mathrm{D}}$, no time history treatment.
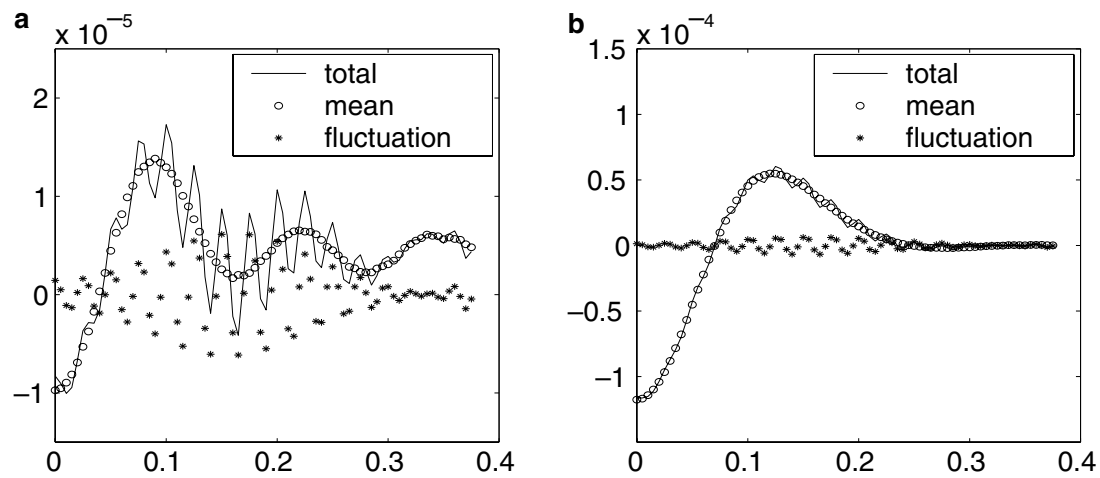

Fig. 10. The source of error in harmonic lattice at $t=150$ : (a) pseudo-spectral MDO-4 scheme and (b) BSM-MLE scheme. 
with the purely fluctuation initial data. This indicates that a balanced accuracy is reached for this case between the coarse grid resolution and the interfacial treatment.

These analyses clearly manifest that the overall accuracy of multiscale computation relies on incorporating a coarse grid scheme and an interfacial condition properly. The pseudo-spectral interfacial conditions are effective in reducing energy interchange between the mean and fluctuation.

\subsection{Nonlinear lattices in one space dimension}

\subsubsection{Anharmonic lattice}

For an anharmonic lattice, we take the same numerical parameters and initial data as for the harmonic lattice, and a nonlinear coefficient $\bar{K}=K h_{a}^{2}=10$. The solution $u(x, t)$ at time $t=50,100,130,150$ is displayed in Fig. 11 for the pseudo-spectral MDO-4 scheme. At $t=50$, the primary wave starts to go across the $\Omega_{\mathrm{C}} / \Omega_{\mathrm{D}}$ interface, whereas fluctuations do not. At $t=100$, fluctuations go across the interface, and minor reflections are observed. We remark that at this moment, the assumption of localized nonlinearity does not hold. Theoretically multiscale methods may fail. However, we still obtain a reasonable resolution. In particular, the primary wave is reproduced well. At $t=150$, the wave has left $\Omega_{\mathrm{D}}$, leaving behind a fairly small amount of energy. Our numerical simulations also show that this energy can be partially reduced if a finer time step size is taken in the MD computation. A longer time history cut-off does not seem to improve the overall accuracy of the computations, as shown in Fig. 12.

\subsubsection{A lattice with the Lennard-Jones potential}

We compute in $\Omega=\left[-200 r_{0}, 200 r_{0}\right]$ with 41 coarse grid points $(p=10)$, and $\Omega_{\mathrm{D}}=\left[-55 r_{0}, 55 r_{0}\right]$ with 111 atoms. We take time steps $\Delta \tau=0.001, \Delta t=0.01$, and initial data
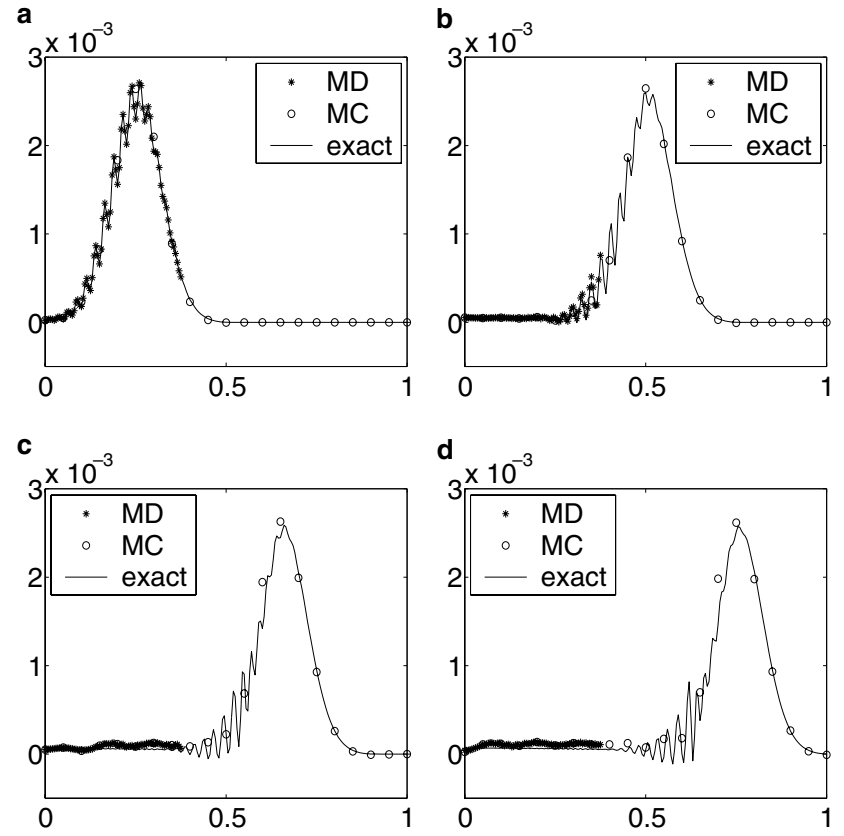

Fig. 11. Anharmonic lattice with $\bar{K}=10$ : (a) $u(x, 50)$; (b) $u(x, 100)$; (c) $u(x, 130)$ and (d) $u(x, 150)$. 

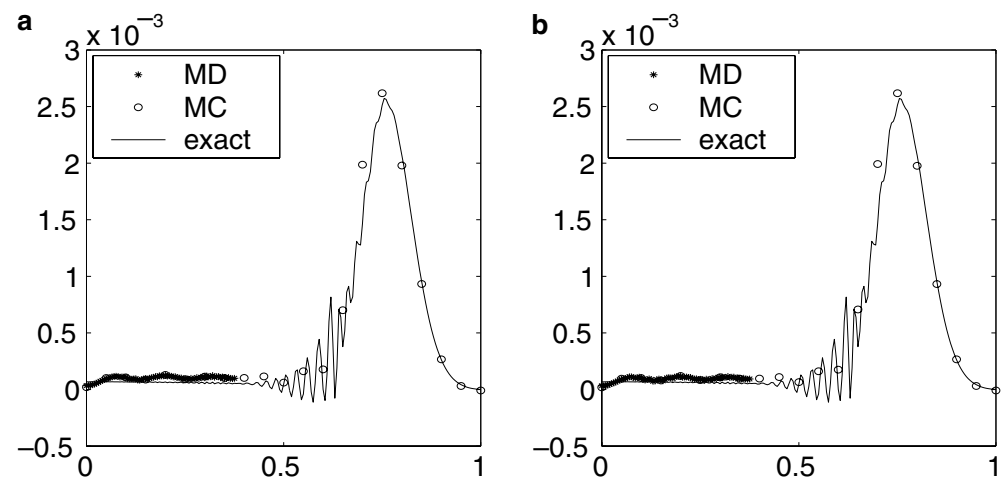

Fig. 12. Anharmonic lattice with $\bar{K}=10$ : (a) longer time history cut-off $T=50$ and (b) finer time step size $\Delta \tau=0.0025$.

$$
u(x)= \begin{cases}0.015 \frac{\mathrm{e}^{-(x / 20)^{2}}-\mathrm{e}^{25}}{1-\mathrm{e}^{25}}(1+0.2 \cos (2 \pi x / 80)) & \text { if }|x|<100 \\ 0, & \text { elsewhere }\end{cases}
$$

The numerical solutions at $t=6,9,12,20$ are displayed in Fig. 13. Basic features are similar to the anharmonic lattice. Agreement with the full MD solution is quite good up to about $t=9$, when the fluctuation partly goes across the $\Omega_{\mathrm{C}} / \Omega_{\mathrm{D}}$ interface. Although the condition required for deriving multiscale methods fails, the numerical schemes still perform very well in a later stage. In particular, the solution in $\Omega_{\mathrm{C}}$ is reproduced faithfully (in the sense of the mean), while the reflections in $\Omega_{\mathrm{D}}$ are fairly small.
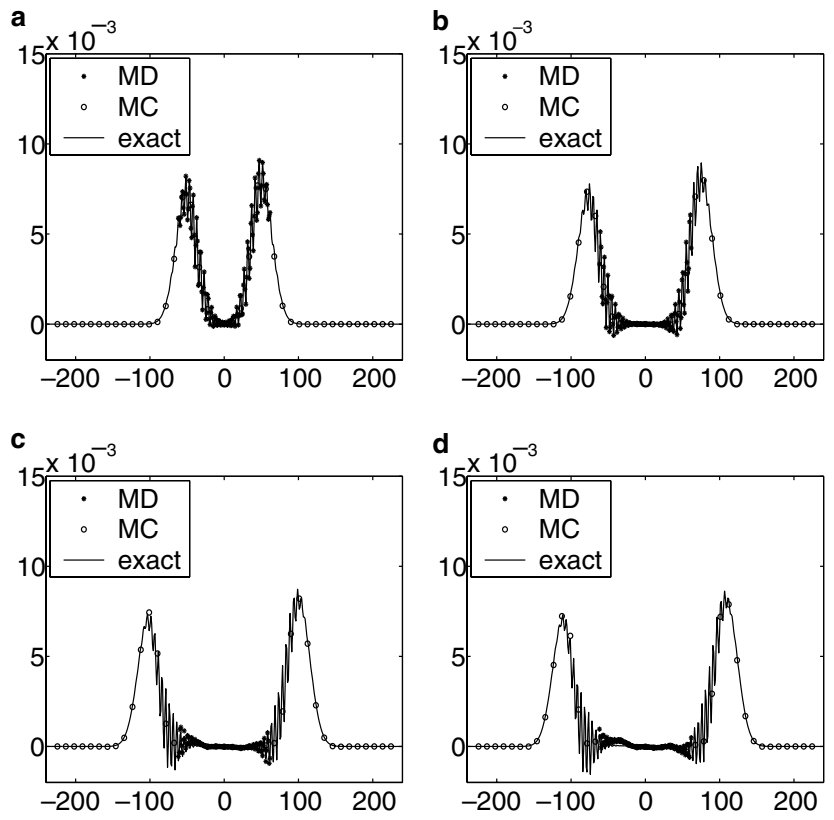

Fig. 13. Lattice with the Lennard-Jones potential: (a) $u(x, 6)$; (b) $u(x, 9)$; (c) $u(x, 12)$ and (d) $u(x, 20)$. 


\subsection{A Slepyan model for fracture in two space dimensions}

We compute in a domain with $257 \times 513$ atoms. The damping coefficient is $b=0.01$. The full MD solution is computed by a full molecular dynamics code, with a time step size $\Delta \tau=0.05$. The coarse grid is set with ratios $p_{x}=p_{y}=8$, in a mesh with $33 \times 65$ grid points. The MD region contains a strip for $j$ between layers 178 and 335, with ghost layers at $j=177$ and 336, respectively. For our multiscale computation, we take $\Delta \tau=0.05$ for MD region, and $\Delta t=0.5$ for the MC update. The time history is kept for $T=20$.

For the external force on upper and lower boundaries, from a systematic study on a triangular lattice, it has been discovered that the crack tip moves only when the external driving force is strong enough, for $C>1$ [17]. For the square lattice, we observe a similar phenomenon. Here we take $C=2$.

Initially, we put a crack between layers 256 and 257, from the first atom to the 48th atom in the $x$-direction. The displacements are piecewise linear. Velocities $\dot{u}_{i j}$ are set to zero uniformly. The initial displacement and subsequent evolution are shown in Fig. 14(a). At an early stage, there is an adjustment of the wave profile, and later on the crack moves ahead.

To quantitatively compare the multiscale computation with the full MD solution, we show the tip position in the two upper subplots of Fig. 15. It remains precisely the same position up to $t \approx 95$. After that, the accumulation of numerical errors starts to make a difference. We further compare the solution at $t=80$, for both the mean displacement over the whole domain and the total displacement in the MD region. The maximum error is about 1 . To get a feeling of how small it is, we note that the snap threshold is 2 , and the displacement around the crack is of the order 50. Fig. 16 shows the comparison at $t=100$. Differences in displacements are almost indiscernible, both in the coarse grid and in the MD region. We remark that with such a thin strip for the MD region (about 1/10 of the total width of $\Omega$ ), the pseudo-spectral multiscale method gives a convergent and accurate resolution of the fracture. On the other hand, it has been noticed that the original BSM method may cause divergent results if the MD domain is not large enough [20].
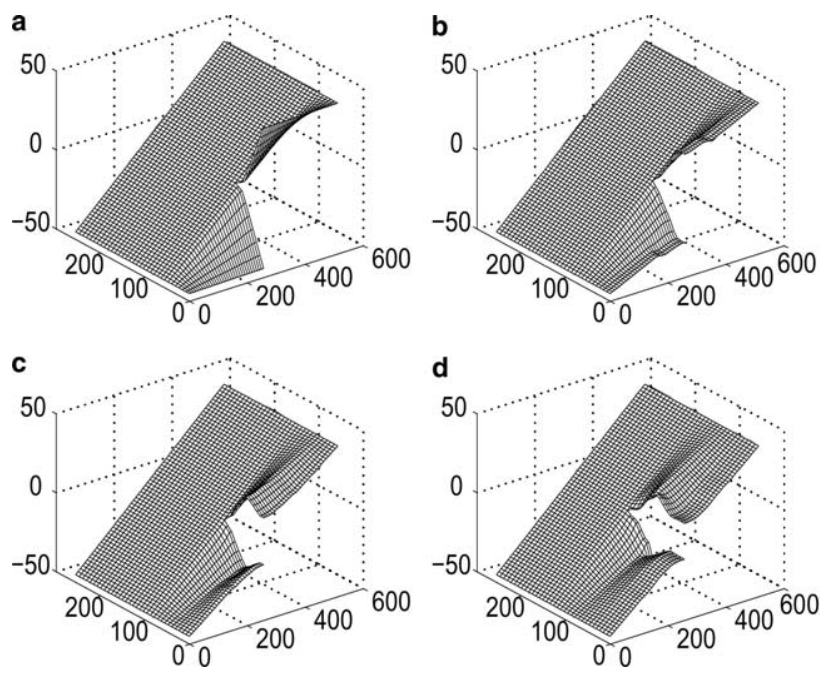

Fig. 14. Coarse grid displacement in Slepyan model by the pseudo-spectral MDO-4 method: (a) $u(x, 0)$; (b) $u(x, 20)$; (c) $u(x, 40)$ and (d) $u(x, 80)$. 
a

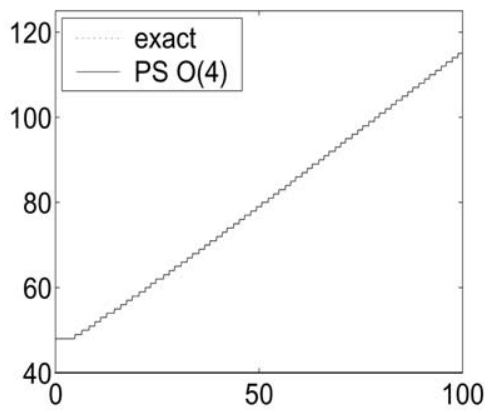

c

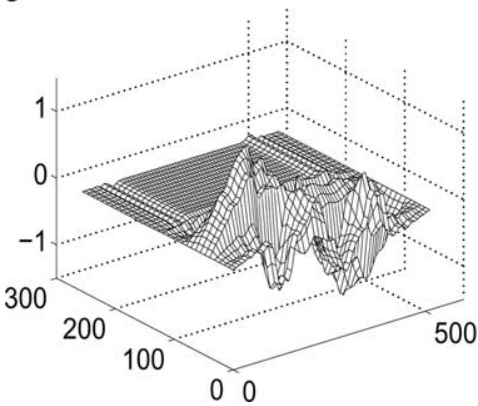

b

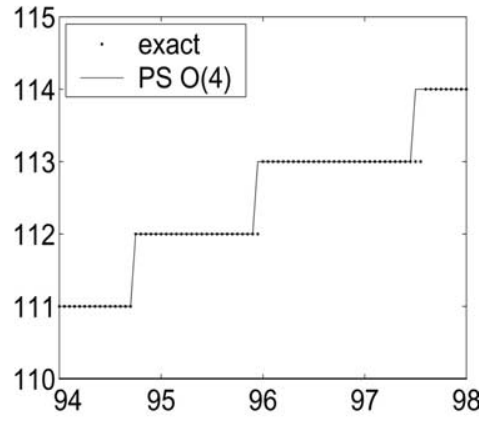

d

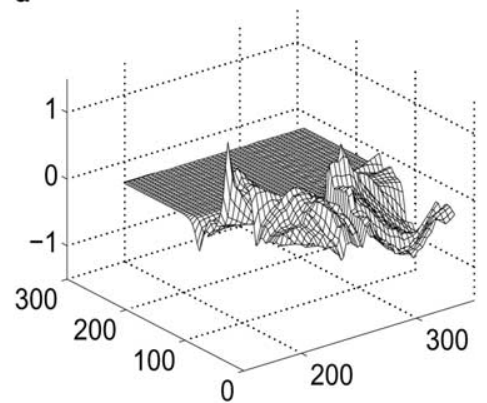

Fig. 15. Error analysis in Slepyan model: (a) crack tip position; (b) crack tip position (local view); (c) error of the mean displacement at $t=80$ and (d) error in the MD region at $t=80$.

a

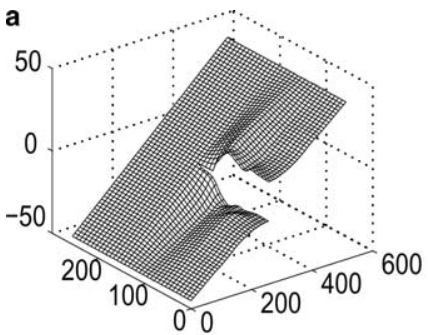

C

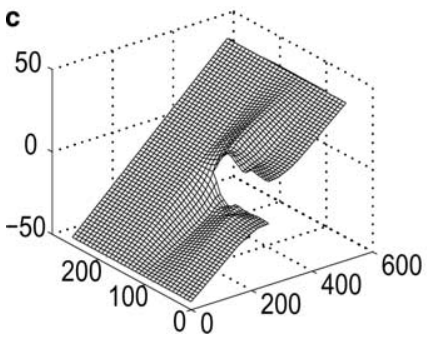

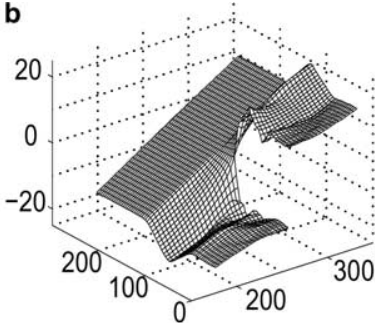

d

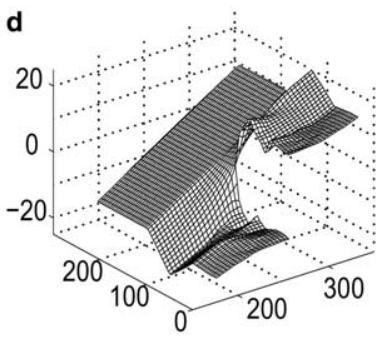

Fig. 16. Displacement in Slepyan model at $t=100$ : (a) coarse grid solution by the pseudo-spectral MDO-4 method; (b) MD solution by the pseudo-spectral MDO-4 method; (c) the full MD solution and (d) the full MD solution in the MD region. 


\section{Discussions}

In this paper, we have proposed a pseudo-spectral multiscale method for simulating complex lattice systems with localized strong nonlinearities.

The key ingredients in the pseudo-spectral multiscale method are the accurate interfacial conditions using the time history treatment, and the coarse grid equations derived by the matching differential operator method. The interfacial conditions are derived with a spectral decomposition of the displacement. We further adopt techniques including the pseudo-spectral approach and the reassignment of the coarse grid displacement in the MD region.

Using these techniques, the pseudo-spectral multiscale method can obtain high order accuracy. Theoretically there is no error incurred in $u_{\mathrm{G}}^{\prime}$ for a linear system, which makes it the first exact condition for multiscale computations. For general systems, the nonlinear effects decrease with the decrement of $\left\|u_{\mathrm{C}}^{\prime}\right\|$. Moreover, a truncation in the displacement time history to a time interval $[t-T, t]$ would introduce an error bounded by $\mathrm{O}\left(T^{-1}\right)$ for the harmonic lattice. Because of the mean-fluctuation decomposition of the displacement, we effectively reduce the energy interchange between these two scales, which is the major error source for this type of approach [25]. Another error source is the pseudo-spectral approximation. Though spectral methods produce no error over individual modes, an aliasing error is introduced by the pseudo-spectral approximation. Nevertheless, we may design coarse grid equations by the matching differential operator method, to any desired order of accuracy for the selected coarse grid. All above-mentioned errors can be made arbitrarily small in principle. This arbitrary error reduction is a major advantage of the pseudo-spectral multiscale method compared with other multiscale methods, which rely heavily on empirical derivations due to the lack of a systematic mathematical analysis.

Other nice features of the pseudo-spectral multiscale method include its robustness, conciseness and clarity due to the absence of a handshaking region. Moreover, apart from the choice of differential operators, the formalism does not include any artificial parameters to tune. It directly applies to quite general situations, including the non-nearest neighbor atom interactions (Appendix B).

Additional cost in the pseudo-spectral multiscale method includes the FFT for spectral decomposition, and the time history convolution. The FFT is performed on the coarse grid only and is known to be very efficient. The convolution is performed at the interface only, which is one dimension lower than the physical space. In addition, we use the time history treatment only for the fluctuations, which has a shorter length scale as well as a shorter time scale. From our experience, when we take several periods in the time history kernel function, the resolution is typically good enough to match the accuracy of the coarse grid computation. The additional cost is rewarded by considerably improved resolution.

There are various aspects for further extension of the pseudo-spectral multiscale method. In particular, the time history treatment is based on the linear approximation of $u_{\mathrm{C}}^{\prime}$ in $\Omega_{\mathrm{C}}$, which is not valid for more general situations. When strong nonlinearity becomes global, we cannot assume $L$ to be time-independent. To resolve this difficulty, we shall compress the time history length properly, and try to use spatial information to extract the time history information for the interfacial atom. Another challenge comes from thermal fluctuations over the whole domain [30]. We plan to model thermal effects by a phonon heat bath. More precisely, we may further introduce a random force to ghost point atoms [13]. The form of the random force is determined by the temperature. A new time history kernel formula is designed with damping applied only to the outgoing waves [26]. Finally, in the current formulation of the pseudo-spectral multiscale method, $u_{\mathrm{C}}^{\prime}$ is relatively small. This is not true, for instance, when a dislocation moves across the interface. There the fluctuation is on the same order as the atomic distance. We shall investigate suitable interfacial treatments in these kinds of applications.

\section{Acknowledgements}

We thank Dr. Eduard Karpov, Dr. Harold Park, Mr. Justin Mach, Ms. Sydney Garstang and Mr. Wuan Luo for stimulating discussions. This research is partially supported by an NSF FRG Grant DMS-0353838 
and an NSF ITR Grant ACI-0204932. S.T. acknowledge partial support of Chinese Special Funds for Major State Basic Research Project Nonlinear Sciences and NSFC under Grant Nos. 90407021 and 10271003. W.K.L. gratefully acknowledge the support of the NSF, and also the NSF-IGERT program. We thank the NSF Summer Institute of Nano Mechanics and Materials, and the Army Research Office (ARO) for their support of this research.

\section{Appendix A. The BSM-MLE method for the harmonic lattice}

The BSM scheme differs from the pseudo-spectral multiscale method mainly by the coarse grid equations and interfacial conditions. We present here a modified version of the BSM scheme for the harmonic lattice [25].

The coarse grid setting is the same as that for the pseudo-spectral multiscale method. Taking $a=\frac{1}{p}[p-1, \ldots, 1,0]^{\mathrm{T}}$, and $b=\frac{1}{p}[1, \ldots, p]^{\mathrm{T}}$, one defines the linear element interpolation matrix

$$
N=\left[\begin{array}{ccccc}
1 & & & & \\
a & b & & & \\
& \ddots & \ddots & & \\
& & & a & b
\end{array}\right] .
$$

The mean displacement and fine fluctuation are defined as $\bar{u}=N d$ and $u^{\prime}=u-\bar{u}$. Here the coarse grid displacement $d=M^{-1} N^{\mathrm{T}} u$ with an effective mass matrix $M=N^{\mathrm{T}} N$. We further define a projection operator $P=N M^{-1} N^{\mathrm{T}}$. It can be shown that

$$
(M \ddot{d})_{J}=\frac{1}{p}\left(d_{J-1}-2 d_{J}+d_{J+1}+u_{(J-1) p}^{\prime}-2 u_{J p}^{\prime}+u_{(J+1) p}^{\prime}\right) .
$$

The original version of BSM uses an approximated equation to make the closure [28]

$$
(M \ddot{d})_{J}=\frac{1}{p}\left(d_{J-1}-2 d_{J}+d_{J+1}\right) .
$$

The MLE (modified linear element) scheme is derived in [25], which yields better coarse grid resolutions. Noticing that $u_{J p}=d_{J}+u_{J p}^{\prime}$, and the right-hand side in $\ddot{u}_{J p}=u_{J p-1}-2 u_{J p}+u_{J p+1}$ approximates the second-order spatial derivative $h_{a}^{2} u_{x x}$ at the $(J p)$ th atom, we approximate it by $\left(d_{J-1}-2 d_{J}+d_{J+1}\right) / p^{2}$. Differentiating (A.2) twice with respect to time, we have

$$
M \frac{\mathrm{d}^{4}}{\mathrm{~d} t^{4}} d=\frac{1}{p^{3}} D^{2} d
$$

It can be shown that away from the boundary, $M$ and $D$ share the same set of eigenvectors, and $\sqrt{M} D=D \sqrt{M}$. This allows us to decompose (A.4) into

$$
\left(\sqrt{M} \frac{\mathrm{d}^{2}}{\mathrm{~d} t^{2}}+\frac{1}{\sqrt{p^{3}}} D\right)\left(\sqrt{M} \frac{\mathrm{d}^{2}}{\mathrm{~d} t^{2}}-\frac{1}{\sqrt{p^{3}}} D\right) d=0 .
$$

The first operator is unstable, therefore irrelevant to the physical problem. An MLE (Modified Linear Element) scheme then follows:

$$
\sqrt{M} \ddot{d}=\frac{1}{\sqrt{p^{3}}} D d \text {. }
$$


For the interfacial conditions, we may first derive the governing equations for $u^{\prime}$ as follows:

$$
\ddot{u}^{\prime}=\left(I-P^{\mathrm{T}}\right) D_{A} u^{\prime} .
$$

Using the Laplace transform, we can show that (assuming $u_{\mathrm{C}}^{\prime}(0)=\dot{u}_{\mathrm{C}}^{\prime}(0)=0$ )

$$
u_{n_{b}+1}^{\prime}=\phi_{1} u_{n_{b}}^{\prime}+\Phi_{\mathrm{G}}\left[I_{\mathrm{C}}\left(D_{A} P-P D_{A}\right) \bar{u}-P_{\mathrm{CD}} D_{\mathrm{D}} u_{\mathrm{D}}^{\prime}-P_{\mathrm{CC}}\left(u_{n_{b}}^{\prime}, 0, \ldots, 0\right)^{\mathrm{T}}\right],
$$

where $I_{\mathrm{C}}=\left[0_{n_{b} \times n_{b}}, I_{\left(n_{a}-n_{b}\right) \times\left(n_{a}-n_{b}\right)}\right], P_{\mathrm{C}}=\left[P_{\mathrm{CD}}, P_{\mathrm{CC}}\right]$ is a submatrix of $P$, and $\Phi_{\mathrm{G}}=\left[\phi_{1}, \ldots, \phi_{n_{a}-n_{b}}\right]$ is the first row of $\mathscr{L}^{-1}\left[\left(s^{2} I-D_{\mathrm{C}}+P_{\mathrm{CC}} D_{\mathrm{C}}+P_{\mathrm{CD}} K_{\mathrm{D}}\right)^{-1}\right]$. Note that $P=N M^{-1} N^{\mathrm{T}}$ is of the order $\mathrm{O}(1 / p)$. When $p$ is big enough, we neglect $P$ in the previous expressions as a first order approximation. This leads to the same interfacial condition as that in the pseudo-spectral multiscale method.

$$
u_{n_{b}+1}^{\prime} \approx \theta * u_{n_{b}}^{\prime} \text {. }
$$

However, because of the different coarse-fine decompositions and the approximations made in ignoring the $P$-related terms, the interfacial condition is not an exact one. This leads to an energy interchange between $\bar{u}$ and $u^{\prime}$ across the interfaces.

\section{Appendix B. A non-nearest neighbor formulation in one dimension}

For a linear lattice with non-nearest neighbor interactions, the coarse grid equation may be derived in the same way as before. We demonstrate how to find the interfacial conditions. To illustrate the idea, we take a semi-infinite chain with the Newton law

$$
\ddot{u}_{n}=\sum_{i=1}^{2} \alpha_{i}\left(u_{n-i}+u_{n+i}\right)-2 \alpha_{0} u_{n}, \quad \alpha_{0}=\alpha_{1}+\alpha_{2} .
$$

We notice that the governing equation for the fine fluctuation $u_{n}^{\prime}$ remains the same. Moreover, we assume $u_{\mathrm{C}}^{\prime}(0)=\dot{u}_{\mathrm{C}}^{\prime}(0)=0$. Now we have two time histories to record, at interfacial atoms labelled $n_{b}-1$ and $n_{b}$. There are also two ghost point atoms, labelled $n_{b}+1, n_{b}+2$. We shall express $u_{n_{b}+1}^{\prime}, u_{n_{b}+2}^{\prime}$ in terms of $u_{n_{b}-1}^{\prime}, u_{n_{b}}^{\prime}$.

In this case, we have

$$
L_{\mathrm{CC}}=\left[\begin{array}{cccccc}
-2 \alpha_{0} & \alpha_{1} & \alpha_{2} & & & \\
\alpha_{1} & -2 \alpha_{0} & \alpha_{1} & \alpha_{2} & & \\
\alpha_{2} & \alpha_{1} & -2 \alpha_{0} & \alpha_{1} & \alpha_{2} & \\
& \ddots & \ddots & \ddots & \ddots & \ddots
\end{array}\right] .
$$

The time history matrix

$$
\Phi=\mathscr{L}^{-1}\left[\left(s^{2} I-L_{\mathrm{CC}}\right)^{-1}\right] .
$$

We will get the first two rows of $\Phi$ to form $\Phi_{\mathrm{G}}$. Because there are only two ghost point atoms, and $u_{\mathrm{C}}^{\prime}(0)=\dot{u}_{\mathrm{C}}^{\prime}(0)=0$, we only need to compute the first two columns in $\Phi_{\mathrm{G}}$. We denote this submatrix as

$$
\Phi_{s}=\left[\begin{array}{ll}
\phi_{11} & \phi_{12} \\
\phi_{21} & \phi_{22}
\end{array}\right]=\mathscr{L}^{-1}\left[\begin{array}{ll}
S_{11} & S_{12} \\
S_{21} & S_{22}
\end{array}\right] .
$$


By a direct computation, we may find the submatrix corresponding to $\Phi_{s}$ as

$$
\begin{aligned}
& S_{11}=\frac{1}{\left(s^{2}+2 \alpha_{0}\right)-\alpha_{1}\left(Z_{1}+Z_{2}\right)-\alpha_{2}\left(Z_{1}^{2}+Z_{1} Z_{2}+Z_{2}^{2}\right)}, \\
& S_{12}=S_{21}=\frac{Z_{1}+Z_{2}}{\left(s^{2}+2 \alpha_{0}\right)-\alpha_{1}\left(Z_{1}+Z_{2}\right)-\alpha_{2}\left(Z_{1}^{2}+Z_{1} Z_{2}+Z_{2}^{2}\right)}, \\
& S_{22}=\frac{\left(s^{2}+2 \alpha_{0}\right) Z_{1} Z_{2}-\alpha_{2} Z_{1}^{2} Z_{2}^{2}}{\alpha_{2}\left[\left(s^{2}+2 \alpha_{0}\right)-\alpha_{1}\left(Z_{1}+Z_{2}\right)-\alpha_{2}\left(Z_{1}^{2}+Z_{1} Z_{2}+Z_{2}^{2}\right)\right]} .
\end{aligned}
$$

Here $Z_{1}, Z_{2}$ are roots with $\left|Z_{1}\right| \leqslant 1,\left|Z_{2}\right| \leqslant 1$ of the algebraic equation

$$
-\alpha_{2} S^{4}-\alpha_{1} S^{3}+\left(s^{2}+2 \alpha_{0}\right) S^{2}-\alpha_{1} S-\alpha_{2}=0 .
$$

It is obvious that if $S$ is a root, so is $1 / S$. therefore $Z_{1}, Z_{2}$ are well defined here.

The interfacial condition then reads

$$
\left[\begin{array}{l}
u_{n_{b}+1}^{\prime} \\
u_{n_{b}+2}^{\prime}
\end{array}\right]=\left[\begin{array}{ll}
\phi_{11} & \phi_{12} \\
\phi_{21} & \phi_{22}
\end{array}\right] *\left[\begin{array}{cc}
\alpha_{2} & \alpha_{1} \\
0 & \alpha_{2}
\end{array}\right]\left[\begin{array}{c}
u_{n_{b}-1}^{\prime} \\
u_{n_{b}}^{\prime}
\end{array}\right] .
$$

\section{Appendix C. Computing the time history kernel in two dimensions}

Except for $i=0$ (damped harmonic lattice), we do not have a closed form of the inverse transform of (38). Therefore we propose to solve it numerically from the ordinary differential equation

$$
\ddot{\theta}_{i}=-b \dot{\theta}_{i}+\theta_{i} * \theta_{i}-\left(2-\zeta_{i}\right) \theta_{i}+\delta(t), \quad \theta_{i}(0)=\dot{\theta}_{i}(0)=0 .
$$

For a small time step $\Delta s$, we define $\theta_{i}^{m}=\theta_{i}(m \Delta s)$. A numerical scheme reads

(1) At $t=0$, take $\theta_{i}^{0}=q_{i}^{0}=a_{i}^{0}=0$;

(2) At $t=\Delta s$, take $\theta_{i}^{1}=\Delta s, q_{i}^{1}=1-b$ and $a_{i}^{1}=-b q_{i}^{1}-\left(2-\zeta_{i}\right) \theta_{i}^{1}$;

(3) For $m \in \mathbb{N}$, take

$$
\begin{aligned}
& \theta_{i}^{m+1}=\theta_{i}^{m}+q_{i}^{m} \Delta s+a_{i}^{m}(\Delta s)^{2} / 2, \\
& q_{i}^{m+1 / 2}=q_{i}^{m}+a_{i}^{m} \Delta s / 2, \\
& a_{i}^{m+1}=-b q_{i}^{m+1 / 2}-\left(2-\zeta_{i}\right) \theta_{i}^{m+1}+\Delta s \sum_{l=1}^{m+1} \theta_{i}^{l} \theta_{i}^{m+2-l}, \\
& q_{i}^{m+1}=q_{i}^{m+1}+a_{i}^{m+1} \Delta s / 2 .
\end{aligned}
$$

Here $q_{i}$ and $a_{i}$ correspond to the derivatives $\dot{\theta}_{i}$ and $\ddot{\theta}_{i}$, respectively. It may be shown that the scheme is of second-order accuracy. In particular, the above choices of $\theta_{i}^{1}, q_{i}^{1}$ and $a_{i}^{1}$ have a error on the order of $\mathrm{O}\left((\Delta s)^{2}\right)$.

\section{References}

[1] S.A. Adelman, J.D. Doll, Generalized Langevin equation approach for atom/solid-surface scattering: collinear atom/harmonic chain model, J. Chem. Phys. 61 (1974) 4242-4245.

[2] F.F. Abraham, J.Q. Broughton, N. Bernstein, E. Kaxiras, Spanning the continuum to quantum length scales in a dynamic simulation of brittle fracture, Europhys. Lett. 44 (1998) 783-787. 
[3] U. Basu, A. Chopra, Perfectly matched layers for time-harmonic elastodynamics of unbounded domains: theory and finite-element implementation, Comput. Methods Appl. Mech. Engrg. 192 (2003) 1337-1375.

[4] J.Q. Broughton, F.F. Abraham, N. Bernstein, E. Kaxiras, Concurrent coupling of length scales: methodology and application, Phys. Rev. B 60 (1999) 2391-2403.

[5] W. Cai, M. de Koning, V.V. Bulatov, S. Yip, Minimizing boundary reflections in coupled-domain simulations, Phys. Rev. Lett. 85 (2000) 3213-3216.

[6] W. Craig, C. Sulem, P.L. Sulem, Nonlinear modulation of gravity waves: a rigorous approach, Nonlinearity 5 (1992) $497-522$.

[7] S.T. O'Connell, P.A. Thompson, Molecular dynamics-continuum hybrid computations: a tool for studying complex fluid flows, Phys. Rev. E 52 (1995) R5792-R5795.

[8] W. E, Z. Huang, A dynamic atomistic-continuum method for simulation of crystalline materials, J. Comput. Phys. 182 (2002) 234-261.

[9] B. Engquist, A. Majda, Rediation boundary conditions for acoustic and elastic calculations, Comm. Pure Appl. Anal. 32 (1979) 313-357.

[10] D. Givoli, J.B. Keller, Non-reflecting boundary conditions for elastic waves, Wave Motion 12 (1990) 261-279.

[11] M.J. Grote, J.B. Keller, Nonreflecting boundary conditions for time-dependent scattering, J. Comput. Phys. 127 (1996) 52-65.

[12] R.L. Higdon, Radiation boundary conditions for dispersive waves, SIAM J. Numer. Anal. 31 (1994) 64-100.

[13] E.G. Karpov, H.S. Park, W.K. Liu, D.L. Dorofeev, On the modelling of chaotic thermal motion in solids, Preprint.

[14] S. Kohlhoff, P. Gumbsch, H.F. Fischmeister, Crack propagation in BCC crystals studied with a combined finite element and atomistic model, Philos. Mag. A 64 (1991) 851-878.

[15] W.K. Liu, E.G. Karpov, H.S. Park, Nano Mechanics and Materials: Theory, Multiscale Methods and Applications, Wiley, 2005.

[16] W.K. Liu, E.G. Karpov, S. Zhang, H.S. Park, An introduction to computational nanomechanics and materials, Comput. Methods Appl. Mech. Engrg. 193 (2004) 1529-1578.

[17] M.P. Marder, Notes on Fracture, http://chaos.ph.utexas.edu/ marder/LesHouches.pdf. 2003.

[18] X.B. Nie, S.Y. Chen, W.N. E, M.O. Robbins, A continuum and molecular dynamics hybrid method for micro- and nano-fluid flow, J. Fluid Mech. 500 (2004) 55-64.

[19] H.S. Park, E.G. Karpov, W.K. Liu, Non-reflecting boundary conditions for atomistic, continuum and coupled atomistic/ continuum simulations, Int. J. Numer. Meth. Engng. 64 (2005) 237-259.

[20] H.S. Park, E.G. Karpov, W.K. Liu, P.A. Klein, The bridging scale for two-dimensional atomistic/continuum coupling, Philos. Mag. 85 (2005) 79-113.

[21] D. Qian, G.J. Wagner, W.K. Liu, A multiscale projection method for the analysis of carbon nanotubes, Comput. Methods Appl. Mech. Engrg. 193 (2004) 1603-1632.

[22] R.E. Rudd, J.Q. Broughton, Concurrent coupling of length scales in solid state systems, Phys. Stat. Sol. 217 (2000) $251-291$.

[23] L.I. Slepyan, M.V. Ayzenberg-Stepanenko, J.P. Dempsey, A lattice model for viscoelastic fracture, Mech. Time-dependent Mater. 3 (1999) 159-203.

[24] E.B. Tadmor, M. Ortiz, R. Phillips, Quasicontinuum analysis of defects in solids, Philos. Mag. A 73 (1996) $1529-1563$.

[25] S. Tang, T.Y. Hou, W.K. Liu, A mathematical framework of the bridging scale method, International J. Numer. Methods Engrg. (in press).

[26] S. Tang, T.Y. Hou, W.K. Liu, Time history treatment for thermal fluctuations (in preparation).

[27] G.J. Wagner, E.G. Karpov, W.K. Liu, Molecular dynamics boundary conditions for regular crystal lattice, Comput. Methods Appl. Mech. Engrg. 193 (2004) 1579-1601.

[28] G.J. Wagner, W.K. Liu, Coupling of atomistic and continuum simulations using a bridging scale decomposition, J. Comput. Phys. 190 (2003) 249-274.

[29] W.W. Wood, F.R. Parker, Monte Carlo equation of state of molecules interacting with the Lennard-Jones potential, J. Chem. Phys. 27 (1957) 720-733.

[30] X. Zhou, H. Chen, M. Iwamoto, Nonunique steady states in the disordered harmonic chain, Phys. Rev. E 66 (2002) 061202. 